\title{
Stability and Isolation Phenomena for Yang-Mills Fields
}

\author{
Jean-Pierre Bourguignon $^{1}$ and H. Blaine Lawson, Jr. ${ }^{2}$ \\ 1 Centre de Mathématiques ${ }^{\star}$, Ecole Polytechnique, F-91128 Palaiseau Cedex, France

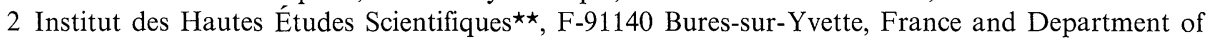 \\ Mathematics, State University of New York, Stony Brook, N.Y. 11790, USA
}

\begin{abstract}
In this article a series of results concerning Yang-Mills fields over the euclidean sphere and other locally homogeneous spaces are proved using differential geometric methods. One of our main results is to prove that any weakly stable Yang-Mills field over $S^{4}$ with group $G=\mathrm{SU}_{2}, \mathrm{SU}_{3}$ or $U_{2}$ is either self-dual or anti-self-dual. The analogous statement for $\mathrm{SO}_{4}$-bundles is also proved. The other main series of results concerns gap-phenomena for YangMills fields. As a consequence of the non-linearity of the Yang-Mills equations, we can give explicit $C^{0}$-neighbourhoods of the minimal Yang-Mills fields which contain no other Yang-Mills fields. In this part of the study the nature of the group $G$ does not matter, neither is the dimension of the base manifold constrained to be four.
\end{abstract}

\section{Introduction and Statement of Results}

The purpose of this article is to prove a series of results concerning Yang-Mills fields over the euclidean sphere and other locally homogeneous spaces by using differential geometric methods. Many of these results were announced in [7].

Our basic set-up is the following. We consider a compact riemannian manifold $M$ and a principal $G$-bundle $P$ over $M$ where $G$ is a compact Lie group. On the space $\mathscr{C}_{P}$ of connections on $G$ we consider the Yang-Mills functional

$$
\mathscr{Y} \mathscr{M}(\nabla)=\frac{1}{2} \int_{M}\left\|R^{\nabla}\right\|^{2},
$$

where $R^{\nabla}$ is the curvature of the connection $\nabla$ in $\mathscr{C}_{P}$ and where the norm is defined in terms of the riemannian metric on $M$ and a fixed $\mathrm{Ad}_{G}$-invariant scalar product on the Lie algebra $g$ of $G$.

Critical points of the smooth function $\mathscr{Y} \mathscr{M}: \mathscr{C}_{P} \rightarrow \mathbb{R}$ are precisely those connections whose curvature tensors are "harmonic". These critical points are

* Laboratoire Associé au C.N.R.S. No. 169

$\star \star$ Research partially supported by Volkswagen Grant and NSF Grant MCS-77-23579 
called Yang-Mills connections and their associated curvature tensors are called Yang-Mills fields. Of particular interest are those Yang-Mills connections which minimize the functional locally, i.e., among nearby connections. At such a connection $\nabla$, the second variation of the functional is non-negative, i.e.,

$$
\left.\frac{d^{2}}{d t^{2}} \mathscr{Y} \mathscr{M}\left(\nabla^{t}\right)\right|_{t=0} \geqq 0
$$

for any smooth family of connections $\nabla^{t},|t|<\varepsilon$, with $\nabla^{0}=\nabla$. Connections with this latter property (1.1) are called stable. This study was motivated by the following result of J. Simons, announced in Tokyo in September of 1977.

Theorem A (J. Simons). There are no weakly stable Yang-Mills fields over the euclidean $n$-sphere $S^{n}$ for $n \geqq 5$.

Recently much effort has been put into studying Yang-Mills fields over $S^{4}$. (This corresponds to studying fields over $\mathbb{R}^{4}$ with certain asymptotic behavior.) In this dimension there are lower bounds for the Yang-Mills functional which depend only on the topology of the bundle. This global lower bound is achieved, for example, if the field $R$ is self-dual, that is if $* R=R$ where $*$ is the Hodge star operator on exterior 2-forms. (This is also true if $* R=-R$, i.e., if the field is antiself-dual.) Self-dual fields are known to exist. In fact there are now explicit constructions of the moduli space of such fields when $G$ is a simple group [2,9]. One of our main results is the following (see Sect. 7).

Theorem B. Any weakly stable Yang-Mills field over $\mathrm{S}^{4}$ with group $\mathrm{G}=\mathrm{SU}_{2}, \mathrm{SU}_{3}$ or $\mathrm{U}_{2}$ is either self-dual or anti-self-dual.

The corresponding theorem for $\mathrm{SO}_{4}$-bundles is proved in Sect. 8. Here the conclusion of self-duality is replaced by one of two-fold self-duality which occurs when the topologically determined lower bound for $\mathscr{Y} \mathscr{M}$ is achieved. In Sect. 10 we prove similar results over general homogeneous spaces of dimension 4.

It is unknown at this moment whether there exist unstable Yang-Mills fields over $S^{4}$.

The instability argument of Simons uses a space $\mathscr{V}$ of conformal vector fields on $S^{n}$ in an essential way. This argument cannot be applied directly in dimension 4 because the functional $\mathscr{Y} \mathscr{M}$ is conformally invariant. In this case we must use the splitting of the field under the *-operator. However, here it suffices to use Killing vector fields. This allows us to extend the results to general homogeneous (orientable) 4-manifolds.

Theorem $\mathbf{B}^{\prime}$. Any weakly stable Yang-Mills field with group $\mathrm{SU}_{2}$ on any compact orientable homogeneous riemannian 4-manifold is either self-dual, or anti-self-dual, or reduces to an abelian field.

The instability argument also breaks down on manifolds $S^{n} / \Gamma$ which are nontrivial quotients of the sphere (real projective space, for example). On each such manifold $S^{n} / \Gamma, n \geqq 4$, we show that stable Yang-Mills fields exist (see Sect. 9). In fact, these fields are strictly stable in the sense that the second variation is strictly positive on a transversal to the orbit of the gauge group (the group of automorphisms of $P$ acting on $\mathscr{C}_{P}$ ). 
Our other main series of results concerns "gap-phenomena" for Yang-Mills fields. This is one of the interesting consequences of the non-linearity of the YangMills equations. To state the results we introduce a scalar product $\langle\cdot, \cdot\rangle$ on $\mathfrak{g}$ by setting $\langle A, B\rangle=-\frac{1}{2} \operatorname{trace}[\varrho(A) \circ \varrho(B)]$ where $\varrho: \mathfrak{g} \rightarrow \mathfrak{s o}_{N}$ is any faithful representation.

Theorem C. Let $R$ be any Yang-Mills field over $S^{n}, n \geqq 3$, which satisfies the pointwise condition

$$
\|R\|^{2} \leqq \frac{1}{2}\left(\begin{array}{l}
n \\
2
\end{array}\right) .
$$

Then either $R \equiv 0$ or $\|R\|^{2} \equiv \frac{1}{2}\left(\begin{array}{l}n \\ 2\end{array}\right)$ and $R$ is parallel.

If $n=3$ or 4 , then either $R \equiv 0$ or $R$ is the curvature of the tangent frame bundle of $S^{n}$ with its Levi-Civita connection.

If $n \geqq 5$, then $R \equiv 0$.

Theorem D. Let $R$ be any Yang-Mills field on $S^{4}$. If $R^{+}$satisfies the pointwise condition $\left\|R^{+}\right\|^{2}<3$, then $R^{+} \equiv 0$. The same statement is true for $R^{-}$.

Note that in Theorems C and D there is no hypothesis concerning the bundle or the group.

Theorem D gives an explicit $C^{0}$-neighborhood of the self-dual (or anti-selfdual) fields on $S^{4}$ in which no other critical points occur. (See [8] for a related result.)

These theorems can be restated in terms of vector bundles with connection.

Theorem $\mathbf{E}$. Let $E$ be any riemannian vector bundle with connection over $S^{n}, n=3$ or 4, whose curvature $R$ is harmonic and satisfies ( $\dagger$ ). Then either $E$ is flat, or $E=S \oplus E_{0}$ where $E_{0}$ is flat and where $S$ is one of the 4-dimensional bundles of tangent spinors with the canonical riemannian connection.

If $E$ is 3-dimensional over $S^{3}$ and if $R$ is harmonic and satisfies the condition $\|R\|^{2}$ $\leqq 3$, then either $E$ is flat or $E=T S^{3}$ with its canonical riemannian connection.

The paper is organized as follows. In Sect. 2 we develop a setting for the YangMills functional using vector bundle theory. In Sect. 3 we establish the BochnerWeitzenböck formulas for harmonic forms with values in a bundle of Lie algebras. In Sect. 4 we examine in detail some algebraic facts specific to dimension 4 , especially in relation to the curvature. In Sect. 5 we establish the Gap Theorems C, $\mathrm{D}$, and $\mathrm{E}$ above. In Sect. 6 we derive several versions of the second variational formula.

The main arguments concerning stability occur in Sect. 7 where Theorems A and $\mathrm{B}$ are proved. Experts interested in these results could begin reading here and refer backwards if and when it becomes necessary. In Sect. 8 we give the examples of stable fields over $S^{n} / \Gamma, n \geqq 4$. We also prove that the index of $T\left(S^{3} / \Gamma\right)$ is exactly one for any $\Gamma$. In Sect. 9 we treat the case of Yang-Mills fields with group $\mathrm{SO}_{4}$ over an oriented 4-manifold. We examine the topological restrictions coming from the Pontryagin and Euler numbers, and we develop the condition of double self- 
duality. The analogue of Theorem $\mathrm{B}$ for $\mathrm{SO}_{4}$-bundles is proved. In Sect. 10 we generalize Theorem B to compact riemannian homogeneous spaces (Theorem B').

We point out that Sect. 5 is completely independent of Sects. 6-10 and may be skipped if the reader wishes.

\section{A Differential Geometric Setting for the Yang-Mills Functional}

For the entire discussion of this paper we fix a compact Lie group $G$ and a principal $G$-bundle $P$ over a compact riemannian $n$-manifold $M$. We also fix a $G$-vector bundle $E=P \underset{\varrho}{\times} \mathbb{R}^{N}$, associated to $P$ by a faithful orthogonal representation $\varrho: G \rightarrow O_{N}$. (Recall that if $P$ is given by transition functions $g_{\alpha \beta}: U_{\alpha} \cap U_{\beta} \rightarrow G$ where $\left\{U_{\alpha}\right\}_{\alpha \in A}$ is an open cover of $M$, then $E$ is given by the transition functions $\varrho \circ g_{\alpha \beta}: U_{\alpha} \cap U_{\beta} \rightarrow O_{N}$.) We shall develop most of our ideas using $E$ rather than $P$ since it is most often the vector bundle that is of central interest. Of course this is also a matter of taste.

Recall that an inner automorphism of $P$ is a $G$-equivariant diffeomorphism of $P$ which projects down to the identity on $M$. The group of all inner automorphisms is called the gauge group of $P$ and will be denoted $\mathscr{G}_{P}$. It can be easily identified with the group of smooth cross-sections of the bundle of groups $G_{P} \equiv P \times G$. Related to $\mathscr{G}_{P}$ is the infinitesimal gauge group or gauge algebra, which will be denoted $\mathfrak{b}_{P}$. It is the Lie algebra of smooth cross-sections of the bundle of Lie algebras $\mathfrak{g}_{P} \equiv P \times \mathfrak{g}$, where $\mathfrak{g}$ is the Lie algebra of $G$. The exponential map exp $: \mathfrak{g} \rightarrow \mathfrak{G}$ induces a natural $\operatorname{map} \exp _{P}: \mathfrak{g}_{P} \rightarrow G_{P}$ and, therefore, a mapping

$$
\exp _{P}: \mathfrak{G}_{P} \rightarrow \mathscr{G}_{P} .
$$

The gauge group can be easily re-expressed in terms of $E$. Let $O_{E}$ be the bundle over $M$ whose fibre at $x$ is the group of orthogonal transformations of $E_{x}$. Let $\mathfrak{s o}_{E}$ be the bundle over $M$ whose fibre at $x$ is the Lie algebra of skew-symmetric transformations of $E_{x}$. Then the representation $\varrho: G \rightarrow O_{N}$ (used to define $E$ ) gives embeddings $G_{P} \rightarrow O_{E}$ and $\mathfrak{g}_{P} \rightarrow \mathfrak{s o}_{E}$ which are homomorphisms on the fibres. We denote the images by $G_{E}$ and $\mathfrak{g}_{E}$ respectively.

Of course, $G_{E} \cong G_{P}$ and $\mathfrak{g}_{E} \cong \mathfrak{g}_{P}$. We may express the gauge group as the space $\mathscr{G}_{E}$ of smooth cross-sections of $G_{E}$. Similarly the gauge algebra is just the space $\mathfrak{G}_{E}$ $=\Omega^{0}\left(\mathfrak{g}_{E}\right)$ of smooth cross-sections of $\mathfrak{g}_{E}$.

Note that the fibre of $G_{E}$ at $\underline{x}$ is just the group of orthogonal transformations of $E_{x}$ which fix the tensors defining the $G$-structure. For example, if $G=\mathrm{SU}_{n}$ and $\varrho: \mathrm{SU}_{n} \rightarrow O_{2 n}$ is the standard homomorphism, then there is a global complex structure $J: E \rightarrow E\left(J^{2} \equiv-1\right)$, and a global nowhere vanishing section $\omega$ of $\Lambda_{\mathbb{C}}^{n} E$. In this case, the sections of $G_{E}$ are those sections of $O_{E}$ which commute with $J$ and fix $\omega$.

There is a similar definition of $g_{E}$. Its fibre at $x$ is just the set of skew-symmetric endomorphisms of $E_{x}$ which, when extended as derivations to the tensor algebra of $E_{x}$, annihilate the tensors defining the $G$-structure. This vector bundle $\mathfrak{g}_{E}$ plays a central role in the theory. 
We now set some notation. Given a smooth vector bundle $F$ over $M$, let $\Omega^{P}(F) \equiv \Gamma\left(\Lambda^{p} T^{*} M \otimes F\right)$ denote the space of exterior differential $p$-forms on $M$ with values in $F$. Note that $\Omega^{0}(F)$ is just the space of smooth cross-sections of $F$.

We want to study the space of connections $\mathscr{C}_{P}$ on $P$, or equivalently on the $G$-vector bundle $E$. Recall that a connection on $P$ is conventionally defined as a $G$-equivariant field of projections $\pi_{P}: T_{p} P \rightarrow \mathscr{V}_{p}, p \in P$, where $\mathscr{V}_{p}$ is tangent space to the fibre through $p$, i.e., the $G$-orbit of $p$. (The kernel of $\pi_{p}$ is the horizontal subspace at $p$.) On the other hand, a connection on $E$ is a linear differential operator $\nabla: \Omega^{0}(E) \rightarrow \Omega^{1}(E)$ such that

$$
\nabla(f \sigma)=d f \otimes \sigma+f \nabla \sigma
$$

for all $f \in C^{\infty}(M), \sigma \in \Omega^{0}(E)$, and such that the natural extension of $\nabla$ to tensor bundles of $E$ annihilates the tensors which define the $G$-structure. These definitions are related as follows. Given $\sigma \in \Omega^{0}(E)$, let $\nabla_{X} \sigma$ denote the evaluation of $\nabla \sigma$ on a tangent vector $X$ at a point $x$. Then

$$
\nabla_{X} \sigma=\tilde{\pi}\left(T_{\sigma}(X)\right),
$$

where $\tilde{\pi}$ is the field of vertical projections on $E$ induced by the trivial extension of $\pi$ to $P \times \mathbb{R}^{N}$. Note that $T \sigma$ maps $T_{x} M$ to $T_{\sigma(x)} E$ and the vertical subspace at $\sigma(x)$ is identified with $E_{x}$ by translation. In this paper we shall work exclusively with connections on $E$.

One can easily see that the difference of two connections $A=\nabla-\nabla^{\prime}$ is an element of $\Omega^{1}\left(\mathfrak{g}_{E}\right)$. Indeed, for any tangent vector field $X, A_{X}=\nabla_{X}-\nabla_{X}^{\prime}$ is a zero order operator, i.e., a bundle homomorphism $A_{X}: E \rightarrow E$ which lies in $\mathrm{g}_{E}$ because both $\nabla$ and $\nabla^{\prime}$ annihilate the tensors defining the $G$-structure. Consequently, the space $\mathscr{C}_{E}$ of connections on $E$ is an affine space with $\Omega^{1}\left(\mathfrak{g}_{E}\right)$ as the vector group of translations. In particular if we fix $\nabla$ in $\mathscr{C}_{E}$ then there is a natural identification

$$
T_{\nabla}\left(\mathscr{C}_{E}\right) \cong \Omega^{1}\left(\mathfrak{g}_{E}\right) \text {. }
$$

To each connection $\nabla$ on $E$ there is associated a curvature 2-form $R^{\nabla}$ in $\Omega^{2}\left(\mathfrak{g}_{E}\right)$ given by the formula

$$
R_{X, Y}^{\nabla} \equiv\left[\nabla_{X}, \nabla_{Y}\right]-\nabla_{[X, Y]}
$$

for tangent vectors $X$ and $Y$.

We note that there is a natural action of the gauge group $\mathscr{G}_{E}$ on the space $\mathscr{C}_{E}$; given $g$ in $\mathscr{G}_{E}$ and $\nabla$ in $\mathscr{C}$ we define

$$
\nabla^{g}=g \circ \nabla \circ g^{-1} .
$$

Here $g$ is considered as an automorphism of $E$. Hence,

$$
\nabla^{g}(\sigma)=g\left(\nabla\left(g^{-1}(\sigma)\right)\right) .
$$

One can easily verify that $\nabla^{g}$ is again a connection on $E$. It is clear from (2.2) that

$$
R^{\nabla^{g}}=g \circ R \circ g^{-1} .
$$


We now observe that the connection $\nabla$ on $E$ induces a natural connection on $\mathfrak{g}_{E}$. Indeed for $\varphi \in \Omega^{0}\left(\mathfrak{g}_{E}\right)$ we define

$$
\nabla(\varphi)=[\nabla, \varphi],
$$

i.e., $\nabla(\varphi)(\sigma)=\nabla(\varphi(\sigma))-\varphi(\nabla \sigma)$ for any section $\sigma$ of $E$. Similarly, the curvature of this connection on $\mathfrak{g}_{E}$ is given by the formula

$$
R_{X, Y}^{\nabla}(\varphi)=\left[R_{X, Y}^{\nabla}, \varphi\right],
$$

where $R^{\nabla}$ on the right denotes the curvature of $E$. The spaces $\Omega^{p}\left(\mathfrak{g}_{E}\right)$ for $p=0,1$, and 2 are clearly of central importance here. For each connection $\nabla$, there are important operators $d^{\nabla}: \Omega^{p}\left(\mathfrak{g}_{E}\right) \rightarrow \Omega^{p+1}\left(\mathfrak{g}_{E}\right), p \geqq 0$, which we now describe.

Let $F$ be any vector bundle over $M$. Then for each linear connection $\nabla$ on $F$ we define an exterior differential $d^{\nabla}: \Omega^{p}(F) \rightarrow \Omega^{p+1}(F), p \geqq 0$, as follows. For each realvalued differential $p$-form $\alpha$ and each section $\sigma$ of $F$, we set

$$
d^{\nabla}(\alpha \otimes \sigma)=(d \alpha) \otimes \sigma+(-1)^{p} \alpha \otimes \nabla \sigma,
$$

and extend the definition to general $\psi \in \Omega^{p}(F)$ by linearity. Note that $d^{\nabla}=\nabla$ on $\Omega^{0}(F)$. It is an easy excercise to prove that

$$
\left(d^{\nabla}\left(d^{\nabla} \sigma\right)\right)_{X, Y}=R_{X, Y}^{\nabla}(\sigma)
$$

for any $\sigma \in \Omega^{0}(F)$. More generally, for any $\psi \in \Omega^{p}(F)$,

$$
\left(d^{\nabla}\left(\mathrm{d}^{\nabla} \psi\right)\right)_{X_{1}, \ldots, X_{p+1}}=\sum_{\sigma \in \mathfrak{E}_{p+2}} R_{X_{\sigma(1)}, X_{\sigma(2)}}^{\nabla}\left(\psi_{X_{\sigma(3)}, \ldots, X_{\sigma(p+2)}}\right) .
$$

Consequently $d^{\nabla} \circ d^{\nabla}=0$ if and only if the bundle $F$ is flat.

Suppose now that $F$ is furnished with an inner product preserved by $\nabla$. We define an inner product in $\Lambda^{p} T_{x}^{*} M \otimes F_{x}$ by setting

$$
\langle\psi, \varphi\rangle=\sum_{i_{1}<\ldots<i_{p}}\left\langle\psi_{e_{i_{1}}, \ldots, e_{i_{p}}}, \varphi_{e_{i_{1}}, \ldots, e_{i_{p}}}\right\rangle,
$$

where $\left(e_{1}, \ldots, e_{n}\right)$ is any orthonormal basis of $T_{x} M$. Integrating this pointwise inner product over $M$ gives an inner product in $\Omega^{p}(F)$. (Integration on $M$ shall always be with respect to the riemannian volume measure.) We then define the operator $\delta^{\nabla}: \Omega^{p+1}(F) \rightarrow \Omega^{p}(F), p \geqq 0$, to be the formal adjoint of the operator $d^{\nabla}$.

The connection $\nabla$ on $F$ together with the Levi-Civita connection $D$ in $\Lambda^{p} T^{*} M$ induces a natural tensor product connection in $\Lambda^{p} T^{*} M \otimes F$ which we again call $\nabla$. Using this connection we have the following simple formulas. For $\varphi \in \Omega^{p}(F)$,

$$
\begin{gathered}
\left(d^{\nabla} \varphi\right)_{X_{0}, \ldots, X_{p}}=\sum_{k=0}^{p}(-1)^{k}\left(\nabla_{X_{k}} \varphi\right)_{X_{0}, \ldots, X_{k}, \ldots, X_{p}}, \\
\left(\delta^{\nabla} \varphi\right)_{X_{1}, \ldots, X_{p-1}}=-\sum_{j=1}^{n}\left(\nabla_{e_{j}} \varphi\right)_{e_{j}, X_{1}, \ldots, X_{p-1}},
\end{gathered}
$$

where $\left(e_{1}, \ldots, e_{n}\right)$ is an orthonormal basis of $T_{x} M$ at the point $x$ in question.

We now introduce an inner product on the bundle $g_{E}$ as follows. Recall that we have $\mathfrak{g}_{E} \subseteq \mathfrak{s o}_{E}$, the bundle of skew-symmetric endomorphisms of $E$. Given two endomorphisms $A$ and $B$ of $E_{x}$, we define

$$
\langle A, B\rangle \equiv \frac{1}{2} \operatorname{trace}\left(A^{t} \circ B\right) .
$$


For the purposes of the theory, any metric on $\mathfrak{g}_{E}$ defined by an $\mathrm{Ad}_{G}$-invariant inner product on $g$ would do. Our choice is adapted to the bundle $E$, in accord with our general emphasis. We shall discuss this inner product in detail at the end of this section.

Given a connection $\nabla \in \mathscr{C}_{E}$ we now have defined a sequence of operators (and their adjoints)

$$
\Omega^{0}\left(\mathfrak{g}_{E}\right) \underset{\delta^{\nabla}}{\stackrel{d^{\nabla}}{\rightleftarrows}} \Omega^{1}\left(\mathfrak{g}_{E}\right) \underset{\delta^{\nabla}}{\stackrel{d^{\nabla}}{\rightleftarrows}} \Omega^{2}\left(\mathfrak{g}_{E}\right) \underset{\delta \nabla}{\stackrel{d^{\nabla}}{\rightleftarrows}} \ldots
$$

Given an element $\sigma \in \Omega^{0}\left(\mathfrak{g}_{E}\right) \cong \mathfrak{5}_{E}$, consider the corresponding curve $g_{t}=\operatorname{Exp}(t \sigma)$ in $\mathscr{G}_{E}$, and note that $\left.(d / d t) \nabla^{g_{t}}\right|_{t=0}=[\nabla \cdot \sigma]=\nabla(\sigma)=d^{\nabla}(\sigma)$. This means that the tangent space to the orbit of the Gauge group at $\nabla$, considered as a subspace of $\Omega^{1}\left(\mathfrak{g}_{E}\right) \cong T_{\nabla} \mathscr{C}_{E}$, is exactly the image, $d^{\nabla}\left(\Omega^{0}\left(\mathfrak{g}_{E}\right)\right)$. Hence, the infinitesimal variations of connection in these directions are infinitesimal variations through gauge equivalent connections. A transversal subspace to image $\left(d^{\emptyset}\right)$ in $\Omega^{1}\left(\mathfrak{g}_{E}\right)$ is given naturally by $\operatorname{ker}\left(\delta^{\downarrow}\right)$. These infinitesimal variations slice across the orbits.

(2.16) Definition. The subspace $\operatorname{ker}\left(\delta^{\emptyset}\right) \subset \Omega^{1}\left(\mathfrak{g}_{E}\right) \cong T_{\nabla} \mathscr{C}_{E}$ is called the space of $i n$ finitesimal deformations of the connection $\nabla$ (Fig. 1)

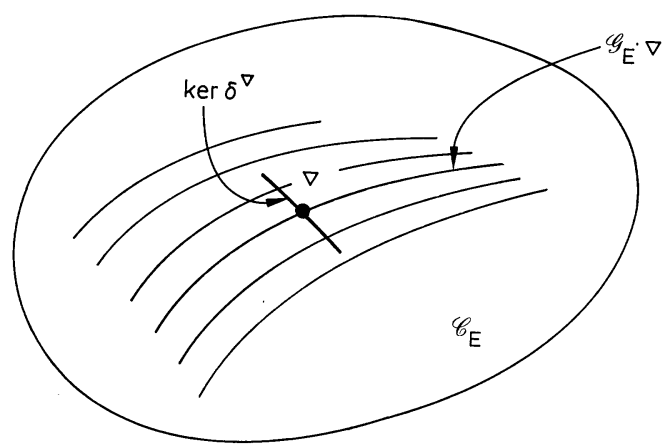

Fig. 1

Note that $\operatorname{ker}\left(\delta^{\emptyset}\right)$ can be thought of as the tangent space of $\mathscr{C} / \mathscr{G}_{E}$ at $[\nabla]$.

Recall that the curvature 2 -form $R$ is an element of $\Omega^{2}\left(\mathfrak{g}_{E}\right)$. This tensor satisfies the Bianchi Identity

$$
d^{\nabla} R^{\nabla}=0,
$$

since $d^{\nabla} R^{\nabla}=d^{\nabla} \circ R^{\nabla}-R^{\nabla} \circ d^{\nabla}=\left(d^{\nabla}\right)^{3}-\left(d^{\nabla}\right)^{3}$. By (2.12) this means that for all tangent vectors $X, Y, Z$

$$
\left(\nabla_{X} R^{\bigvee}\right)_{Y, Z}+\left(\nabla_{Y} R^{\bigvee}\right)_{Z, X}+\left(\nabla_{Z} R^{\bigvee}\right)_{X, Y}=0
$$

We now present the concepts central to our paper.

(2.19) Definition. The function $\mathscr{Y} \mathscr{M}: \mathscr{C}_{E} \rightarrow \mathbb{R}$ defined by

$$
\mathscr{Y} \mathscr{M}(\nabla)=\frac{1}{2} \int_{M}\left\|R^{\nabla}\right\|^{2}
$$


is called the Yang-Mills functional. A critical point $\nabla \in \mathscr{C}_{E}$ of the Yang-Mills functional is called a Yang-Mills connection. The curvature $R^{\nabla}$ of a Yang-Mills connection is called a Yang-Mills field.

Note that by (2.5) the functional $\mathscr{Y} \mathscr{M}$ is clearly invariant under the gauge group $\mathscr{G}_{E}$ acting on $\mathscr{C}_{E}$. It is, in fact, invariant under an "enlarged gauge group" which we shall discuss presently.

Fix $\nabla \in \mathscr{C}_{E}$ and consider a smooth family of connections $\nabla^{t},-\varepsilon<t<\varepsilon$, such that $\nabla^{0}=\nabla$. The statement that $\nabla$ is a critical point of $\mathscr{Y} \mathscr{M}$ means simply that $\left.(d / d t) \mathscr{Y} \mathscr{M}\left(\nabla^{t}\right)\right|_{t=0}=0$ for all such families. We write

$$
\nabla^{t}=\nabla+A^{t},
$$

where $A^{t} \in \Omega^{1}\left(\mathfrak{g}_{E}\right)$ for $|t|<\varepsilon$ and $A^{0}=0$. The corresponding curvature is given by

$$
R^{\nabla^{t}}=R^{\nabla}+\mathrm{d}^{\nabla} \mathrm{A}^{t}+\frac{1}{2}\left[A^{t} \wedge A^{t}\right]
$$

where we define the bracket of $\mathfrak{g}_{E}$-valued 1-forms $\varphi$ and $\psi$ by the formula $[\varphi \wedge \psi]_{X, Y}=\left[\varphi_{X}, \psi_{Y}\right]-\left[\varphi_{Y}, \psi_{X}\right]$. The following basic fact is an immediate consequence of (2.20) and the definition of the adjoint of an operator.

(2.21) Theorem. The first variation of the Yang-Mills functional is given by the formula

$$
\left.\frac{d}{d t} \mathscr{Y} \mathscr{M}\left(\nabla^{t}\right)\right|_{t=0}=\int_{M}\left\langle\delta^{\nabla} R^{\nabla}, \mathrm{B}\right\rangle
$$

where

$$
B=\left.\frac{d}{d t} \nabla^{t}\right|_{t=0}
$$

Consequently, $\nabla$ is a Yang-Mills connection if and only if

$$
\delta^{\nabla} R^{\nabla}=0 .
$$

This equation is non-linear in $\nabla$. Using the Bianchi Identity (2.18) and the compactness of $M$ we see that (2.22) is equivalent to the condition that

$$
\Delta^{\nabla} R^{\nabla}=0
$$

where

$$
\Delta^{\nabla} \equiv d^{\nabla} \delta^{\nabla}+\delta^{\nabla} d^{\nabla}
$$

is the generalized Hodge-deRham Laplacian for vector bundle valued exterior $p$-forms. Any form $\varphi \in \Omega^{p}\left(\mathfrak{g}_{E}\right)$ satisfying the equation $\Delta^{\nabla} \varphi=0$ will be called harmonic. Thus, Yang-Mills connections are connections with harmonic curvature.

There is another second order operator $\nabla^{*} \nabla$, called the rough Laplacian, defined on $\mathfrak{g}_{E}$-valued differential forms. It is given by the formula

$$
\nabla^{*} \nabla \varphi=-\sum_{j=1}^{n}\left(\nabla_{e_{j}, e_{j}}^{2} \varphi\right),
$$


where

$$
\nabla_{X, Y}^{2} \equiv \nabla_{X} \nabla_{Y}-\nabla_{\left(D_{X} Y\right)}
$$

is the invariantly defined Hessian operator. The operator $\nabla^{*} \nabla$ is symmetric and non-negative. Its kernel is the space of parallel forms. The operators $\Delta^{\nabla}$ and $\nabla^{*} \nabla$ have the same principal symbol, and their difference is of zero order. The relationship between these operators will be crucial in this paper.

In the case that $M$ is an oriented manifold, we may introduce the Hodge staroperator $*: \Omega^{p}\left(\mathfrak{g}_{E}\right) \rightarrow \Omega^{n-p}\left(\mathfrak{g}_{E}\right)$ into our formulas. In particular we have that on $\Omega^{p}\left(\mathfrak{g}_{E}\right)$

$$
(-1)^{p+1} d^{\nabla} * * * \delta^{\nabla}
$$

Moreover, the Yang-Mills functional can be rewritten as

$$
\mathscr{Y} \mathscr{M}(\nabla)=\frac{1}{2} \int_{M}\left\langle R^{\nabla} \wedge * R^{\nabla}\right\rangle,
$$

where the notation $\langle\varphi \wedge \psi\rangle$ for $\varphi, \psi \in \Lambda^{*} \mathrm{~T}^{*} M \otimes \mathfrak{g}_{E}$ means exterior product on $\Lambda^{*} T^{*} M$ tensored with the interior product on $\mathfrak{g}_{E}$.

We now return briefly to the inner product defined on the bundle $\mathfrak{s o}_{E}$ (and thereby on $\mathfrak{g}_{E}$ ) by formula (2.14). Recall that there is a natural bundle isomorphism $\Lambda^{2} E \stackrel{\approx}{\longrightarrow} \mathfrak{s o}_{E}$ determined by the requirement that

$$
(u \wedge v)(w)=\langle u, w\rangle v-\langle v, w\rangle u
$$

for $u, v, w \in E_{x}$. In the metric (2.11), the elements $\left\{\varepsilon_{i} \wedge \varepsilon_{j}\right\}_{i<j}$ form an orthonormal basis of $\left(\mathfrak{s o}_{E}\right)_{x}$ whenever $\left(\varepsilon_{1}, \ldots, \varepsilon_{N}\right)$ is an orthonormal basis of $E_{x}$. This scalar product is related to the standard Killing form $K$ on the bundle of Lie algebras $\mathfrak{s o}_{E}$ by the formula $K(A, B)=-2(N-2)\langle A, B\rangle$.

(2.30) Lemma. The norm $\|\cdot\|$ induced by the inner product (2.14) has the property that

$$
\|[A, B]\| \leqq \sqrt{2}\|A\|\|B\|
$$

for all $A, B$ with equality if and only if the pair $A, B$ is orthogonally equivalent to the pair of Pauli matrices (Fig. 2)
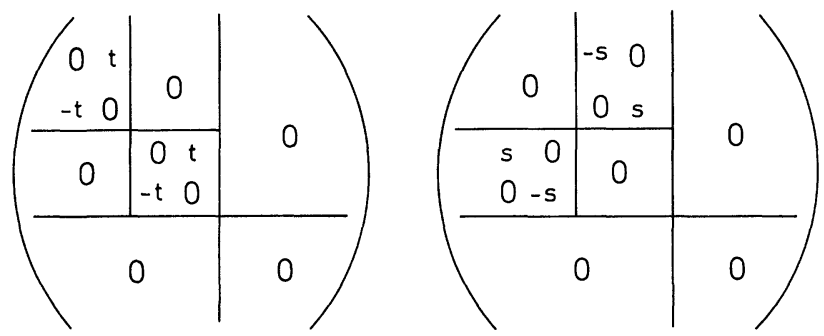

Fig. 2 
Proof. Maximize the function $(A, B) \mapsto\|[A, B]\|^{2}$ under the constraints $\|A\|^{2}$ $=\|B\|^{2}=1$. At any critical point, we find that

$$
\begin{aligned}
& (\operatorname{ad} B)^{2} A=\lambda A \\
& (\operatorname{ad} A)^{2} B=\mu B
\end{aligned}
$$

for multipliers $\lambda$ and $\mu$. An easy calculation shows that $\lambda=\mu=-\|[\mathrm{A}, \mathrm{B}]\|^{2}$. Put $B$ in (skew) diagonal form and examine the first equation above. The remainder of the argument is straightforward and we omit the details.

(2.31) Note. Recall that in $\mathfrak{s o}_{3} \cong \mathbb{R}^{3}$ the Lie algebra product is equivalent to the classical cross-product in $\mathbb{R}^{3}$. Thus when $\operatorname{dim}(E)=3$, the inequality $(2.30)$ can be improved to: $\|[A, B]\| \leqq\|A\|\|B\|$, with equality iff $A \perp B$.

We finish this section with a word concerning the enlarged gauge group. We define an outer automorphism of $P$ to be any $G$-equivariant diffeomorphism of $P$, and we denote the group of all outer automorphisms by $\operatorname{Diff}_{G}(P)$. Every outer automorphism $f: P \rightarrow P$ projects to a diffeomorphism $\pi(f): M \rightarrow M$. This yields an exact sequence of groups :

$$
1 \longrightarrow \mathscr{G}_{P} \longrightarrow \operatorname{Diff}_{G}(P) \stackrel{\pi}{\longrightarrow} \operatorname{Diff}(M) .
$$

Given a connection on $P$ and a vector field $X$ on $M$, we have a unique lifting of $X$ to a horizontal vector field $\tilde{X}$ on $P$. Let $\varphi_{t}, \tilde{\varphi}_{t}$ be the 1-parameter groups of diffeomorphisms generated by $X$ and $\tilde{X}$ respectively. Since $\tilde{X}$ is $G$-invariant, we have that $\tilde{\varphi}_{t} \circ g=g \circ \tilde{\varphi}_{t}$ for $g \in G$ and for all $t$. Hence, $\tilde{\varphi}_{t} \in \operatorname{Diff}_{G}(P)$ and clearly $\pi\left(\tilde{\varphi}_{t}\right)$ $=\varphi_{t}$ for all $t$. From the work of Thurston [15] we know that the diffeomorphisms belonging to flows generate the identity component $\operatorname{Diff}^{0}(M)$. Hence, the image of $\pi$ contains this group and we have the short exact sequence.

$$
1 \longrightarrow \mathscr{G}_{P} \longrightarrow \operatorname{Diff}_{G}^{0}(P) \longrightarrow \operatorname{Diff}^{0}(M) \longrightarrow 1
$$

Any connection on $P$ determines a splitting of the sequence of vector spaces $(2.33)_{\mathrm{e}}^{\prime}$ obtained by differentiating (2.33) at the identity. It is given by the correspondence $X \mapsto \tilde{X}$ constructed above. Conversely, each splitting of $(2.33)_{\mathrm{e}}^{\prime}$ determines a connection on $P$. Hence, a connection could be defined as such a splitting.

The entire group $\operatorname{Diff}_{G}(P)$ obviously acts on the space $\mathscr{C}_{P}$ of connections. A subgroup which always preserves the Yang-Mills functional is the group $\tilde{\mathscr{G}}_{P}=\pi^{-1}\left(I_{M}\right)$ where $I_{M}$ denotes the group of isometries of $M$ when $n \neq 4$ and the group of all conformal transformations of $M$ when $n=4$. We call $\tilde{\mathscr{G}}_{P}$ the enlarged gauge group of $P$.

It is interesting to consider the variations of connection that come from $\operatorname{Diff}_{G}(P)$. Fix $\nabla \in \mathscr{C}$ and consider a vector field $X$ on $M$. Then the infinitesimal variation of connection $B=\left.\frac{d}{d t} \nabla^{t}\right|_{t=0}$, where $\nabla^{t}=\tilde{\varphi}_{-t}^{*} \nabla$ and where $\tilde{\varphi}_{t}$ is associated to $\tilde{X}$ as above, is given by the formula

$$
B=i_{X} R^{\nabla}
$$


where $i_{X}$ denotes contraction with $X$. For a quick proof of this, let $\pi: T P \rightarrow \mathscr{V}$ be the $G$-equivariant field of projections along the horizontal subspaces $\mathscr{H}$ onto the field of vertical subspaces $\mathscr{V}$. The tensor $B$ is nothing but $\left.\frac{d}{d t} \tilde{\varphi}_{t}^{*} \pi\right|_{t=0}$ viewed as a linear map from $\mathscr{H}$ to $\mathscr{V}$ where each $\mathscr{V}_{p}$ is canonically identified with $\mathfrak{g}$ (and with the corresponding fibre in $\mathfrak{g}_{P}$ ). Of course $\left.\frac{d}{d t} \tilde{\varphi}_{t}^{*} \pi\right|_{t=0}=-\mathfrak{S}_{\tilde{X}}(\pi)$ where $\mathfrak{S}_{\tilde{X}}$ denotes the Lie derivative. Furthermore for any tangent vector field $Y$ on $M$ let $\tilde{Y}$ denote the horizontal lift to $P$ and note that

$$
\begin{aligned}
\left(\mathfrak{Q}_{\tilde{X}} \pi\right)(\tilde{Y}) & =\mathfrak{Q}_{\tilde{X}}(\pi \tilde{Y})-\pi\left(\mathfrak{S}_{\tilde{X}} \tilde{Y}\right) \\
& =[\tilde{X}, \pi \tilde{Y}]-\pi([\tilde{X}, \tilde{Y}]) \\
& =-\pi([\tilde{X}, \tilde{Y}]) \\
& \cong-R_{X, Y}=-\left(i_{X} R\right)_{Y} .
\end{aligned}
$$

These last remarks add insight to the discussion of Sect. 7.

\section{The Bochner-Weitzenböck Formulas for Lie Algebra-Bundle-Valued Forms}

The purpose of this section is to establish formulas relating the Hodge-de Rham Laplacian and the rough Laplacian of $\mathfrak{g}_{E}$-valued 1- and 2-forms. They involve both the curvature of the riemannian base manifold and of the bundle with connection. We deduce from them an algebraic expression of the rough Laplacian of a harmonic $\mathfrak{g}_{E}$-valued $p$-form ( $p=1$ or 2 ). These formulas are of fundamental importance in the theory.

To begin we define a basic (zero-order) operator $\mathfrak{R}^{P}: \Omega^{1}\left(\mathfrak{g}_{E}\right) \rightarrow \Omega^{1}\left(\mathfrak{g}_{E}\right)$ by setting

$$
\mathfrak{S}^{\nabla}(\varphi)_{X} \equiv \sum_{j=1}^{n}\left[R_{e_{j}, X}^{\nabla}, \varphi_{e_{j}}\right]
$$

where $R^{\nabla}$ denotes the curvature of the connection $\nabla$ on $E$ and where $\left(e_{1}, \ldots, e_{n}\right)$ is an orthonormal basis of the tangent space $T_{x} M$ at the point $x$ in question. Recall that the Ricci transformation Ric: $T_{x} M \rightarrow T_{x} M$ is defined by

$$
\operatorname{Ric}(X)=\sum_{j=1}^{n} R_{X, e_{j}} e_{j},
$$

where $R$ here denotes the riemannian curvature tensor. Given $\varphi$ in $\Omega^{1}\left(\mathfrak{g}_{E}\right)$ we define

$$
(\varphi \circ \operatorname{Ric})_{X}=\varphi_{\operatorname{Ric}(X)} .
$$

We recall that on the standard sphere $\operatorname{Ric}(X)=(n-1) X$.

(3.2) Theorem. For any $\varphi$ in $\Omega^{1}\left(\mathfrak{g}_{E}\right)$, we have

$$
\Delta^{\nabla} \varphi=\nabla^{*} \nabla \varphi+\varphi \circ \operatorname{Ric}+\boldsymbol{\Omega}^{\nabla}(\varphi) .
$$

Proof. Fix a point $x$ in $M$ and choose $X, e_{1}, \ldots, e_{n}$ in $T_{x} M$ so that $\left(e_{1}, \ldots, e_{n}\right)$ is an orthonormal basis. Extend $X$ to a local vector field and $\left(e_{1}, \ldots, e_{n}\right)$ to a local 
orthonormal frame field so that $(D X)(x)=\left(D e_{1}\right)(x)=\ldots=\left(D e_{n}\right)(x)=0$. Then, at the point $x$,

$$
\begin{aligned}
\left(d^{\nabla} \delta^{\nabla} \varphi\right)_{X} & =\nabla_{X}\left(\delta^{\nabla} \varphi\right)=-\nabla_{X}\left(\sum_{j=1}^{n}\left(\nabla_{e_{j}} \varphi\right) e_{j}\right) \\
& =-\sum_{j=1}^{n}\left(\nabla_{X, e_{j}}^{2} \varphi\right) e_{j}
\end{aligned}
$$

and

$$
\begin{aligned}
\left(\delta^{\nabla} d^{\nabla} \varphi\right)_{X} & =-\sum_{j=1}^{n}\left(\nabla_{e_{j}} d^{\nabla} \varphi\right)_{e_{j}, X} \\
& =-\sum_{j=1}^{n} \nabla_{e_{j}}\left\{\left(\nabla_{e_{j}} \varphi\right)_{X}-\left(\nabla_{X} \varphi\right)_{e_{j}}\right\} \\
& =-\sum_{j=1}^{n}\left\{\left(\nabla_{e_{j}, e_{j}}^{2} \varphi\right)_{X}-\left(\nabla_{e_{j}, X}^{2} \varphi\right)_{e_{j}}\right\}
\end{aligned}
$$

Adding (3.3) and (3.4) we find that

$$
\left(\Delta^{\nabla} \varphi\right)_{X}=\left(\nabla^{*} \nabla \varphi\right)_{X}+\sum_{j=1}^{n}\left(R_{e_{j}, X}^{\nabla} \varphi\right)_{e_{j}}
$$

(here we keep the notation $R^{\nabla}$ for the curvature of the connection on the bundle $T^{*} M \otimes \mathfrak{g}_{E}$ induced by the Levi-Civita connection $D$ on the base and the connection $\nabla$ on the bundle $E$ ).

The operator $R^{\nabla}$ acts as a derivation. Hence $\left(R_{X, Y}^{\nabla} \varphi\right)_{Z}=\left[R_{X, Y}^{\nabla}, \varphi_{Z}\right]-\varphi_{R_{X, Y} Z}$. Applying this formula to the second term in (3.5) gives the result.

(3.6) Corollary. If $\varphi$ is a $\mathfrak{g}_{E}$-valued $\nabla$-harmonic 1-form on the standard sphere $S^{n}$, then

$$
\nabla^{*} \nabla \varphi=-(n-1) \varphi-\mathfrak{R}^{\nabla}(\varphi) .
$$

Similar calculations can be carried out for any $p$-form. Of particular interest here is the case $p=2$. In analogy with (3.1) we define $\mathfrak{H}^{\nabla}: \Omega^{2}\left(\mathfrak{g}_{E}\right) \rightarrow \Omega^{2}\left(\mathfrak{g}_{E}\right)$ by

$$
\mathfrak{S}^{\nabla}(\varphi)_{X, Y} \equiv \sum_{j=1}^{n}\left\{\left[R_{e_{j}, X}^{\nabla}, \varphi_{e_{j}, Y}\right]-\left[R_{e_{j}, Y}^{\nabla}, \varphi_{e_{j}, X}\right]\right\} .
$$

For a linear map $\omega$ on 2-vectors we define

$$
(\varphi \circ \omega)_{X, Y}=\frac{1}{2} \sum_{j=1}^{n} \varphi_{e_{J}, \omega_{X, Y} e_{j}}
$$

(this is indeed the composition of $\omega$ and $\varphi$ viewed as maps from $\Lambda^{2} T M$ to $\mathfrak{g}_{E}$ ). Notice that with our convention $\varphi_{X, Y}=\varphi(X \wedge Y)$.

Notice then that the extension of the Ricci transformation to 2-forms as Ric $\wedge I$ is given by

$$
(\operatorname{Ric} \wedge I)_{X, Y}=\operatorname{Ric}(X) \wedge Y+X \wedge \operatorname{Ric}(Y) .
$$

(3.10) Theorem. For any $\varphi$ in $\Omega^{2}\left(\mathfrak{g}_{E}\right)$

$$
\Delta^{\nabla} \varphi=\nabla^{*} \nabla \omega+\varphi \circ(\operatorname{Ric} \wedge g+2 R)+\mathfrak{R}^{\nabla}(\varphi) .
$$


Proof. Fix $x$ in $M$. Choose $X, Y, e_{1}, \ldots, e_{n}$ in $T_{x} M$ and extend them as in the proof of Theorem (3.2). Then at the point $x$

$$
\begin{aligned}
\left(d^{\nabla} \delta^{\nabla} \varphi\right)_{X, Y} & =\left(\nabla_{X} \delta^{\nabla} \varphi\right)_{Y}-\left(\nabla_{Y} \delta^{\nabla} \varphi\right)_{X} \\
& =-\nabla_{X}\left(\sum_{j=1}^{n}\left(\nabla_{e_{j}} \varphi\right)_{e_{j}, Y}\right)+\nabla_{Y}\left(\sum_{j=1}^{n}\left(\nabla_{e_{j}} \varphi\right)_{e_{j}, X}\right) \\
& =-\sum_{j=1}^{n}\left\{\left(\nabla_{X, e_{j}}^{2} \varphi\right)_{e_{j}, Y}-\left(\nabla_{Y, e_{j}}^{2} \varphi\right)_{e_{j}, X}\right\}
\end{aligned}
$$

and

$$
\begin{aligned}
\left(\delta^{\nabla} d^{\nabla} \varphi\right)_{X, Y} & =-\sum_{j=1}^{n}\left(\nabla_{e_{j}} d^{\nabla} \varphi\right)_{e_{j}, X, Y} \\
& =-\sum_{j=1}^{n} \nabla_{e_{j}}\left\{\left(\nabla_{e_{j}} \varphi\right)_{X, Y}+\left(\nabla_{Y} \varphi\right)_{e_{j}, X}+\left(\nabla_{X} \varphi\right)_{Y, e_{j}}\right\} \\
& =-\sum_{j=1}^{n}\left\{\left(\nabla_{e_{j}, e_{j}}^{2} \varphi\right)_{X, Y}+\left(\nabla_{e_{j}, Y}^{2} \varphi\right)_{e_{j}, X}-\left(\nabla_{e_{j}, X}^{2} \varphi\right)_{e_{j}, Y}\right\} .
\end{aligned}
$$

Combining (3.11) and (3.12) we find that

$$
\left(\Delta^{\nabla} \varphi\right)_{X, Y}=\left(\nabla^{*} \nabla \varphi\right)_{X, Y}+\sum_{j=1}^{n}\left\{\left(R_{e_{j}, X}^{\nabla} \varphi\right)_{e_{j}, Y}-\left(R_{e_{j}, Y}^{\nabla} \varphi\right)_{e_{j}, X}\right\}
$$

By using the identity

$$
\left(R_{X, Y}^{\nabla} \varphi\right)_{Z, W}=\left[R_{X, Y}^{\nabla}, \varphi_{Z, W}\right]-\varphi_{R_{X, Y} Z, W}-\varphi_{Z, R_{X}, Y},
$$

Equation (3.13) can be expressed as

$$
\begin{aligned}
\left(\Delta^{\nabla} \varphi\right)_{X, Y}= & \left(\nabla^{*} \nabla \varphi\right)_{X, Y}+\mathfrak{R}^{\nabla}(\varphi)_{X, Y}+\varphi_{\mathrm{Ric}(X), Y} \\
& -\sum_{j=1}^{n} \varphi_{e_{j}, R_{e_{j}, X} Y}-\varphi_{\mathrm{Ric}(Y), X}+\sum_{j=1}^{n} \varphi_{\boldsymbol{e}_{j}, \boldsymbol{R}_{e_{j}, Y} X} .
\end{aligned}
$$

By using the first Bianchi identity

$$
R_{X, Y} Z+R_{Y, Z} X+R_{Z, X} Y=0
$$

together with the Definitions (3.8) and (3.9), we get the expected relation.

Remarks. i) The sign in the formula of Theorem (3.10) in front of $\mathfrak{R}$ may change with conventions.

ii) This way of expressing the formula has the advantage of incorporating the influence of the curvature of the base manifold into one term. This is especially interesting in dimension 4, where one can check (cf. [5]), by decomposing the riemannian curvature into irreducible components under the action of $O_{4}$ or $\mathrm{SO}_{4}$, that $\operatorname{Ric} \wedge I+2 R$ does not involve the Ricci traceless part of the riemannian curvature. For an application see Sect. 5.

(3.14) Corollary. If $\varphi$ is a $\mathfrak{g}_{E}$-valued harmonic 2-form on the standard sphere $S^{n}$, then

$$
\nabla^{*} \nabla \varphi=-2(n-2) \varphi-\mathfrak{S}^{\nabla}(\varphi) .
$$


Proof. On the standard spheres $S^{n}$, we have

$$
R_{X, Y} Z=-(X \wedge Y)(Z)=\langle Y, Z\rangle X-\langle X, Z\rangle Y .
$$

Therefore,

$$
(\operatorname{Ric} \wedge I+2 R)_{X, Y}=2(n-2) X \wedge Y .
$$

We conclude this section with an elementary fact that will be of use in Sect. 10.

(3.15) Proposition. Suppose $\varphi \in \Omega^{2}\left(\mathfrak{g}_{E}\right)$ takes values in a 1-dimensional subbundle of $\mathfrak{g}_{E}$, i.e., suppose $\varphi=f \otimes \sigma$ where $f$ is a scalar-valued 2-form and where $\sigma$ is a section of $\mathfrak{g}_{E}$ with $\|\sigma\|^{2} \equiv 1$. Suppose further that $\mathfrak{S}^{\Gamma}(\varphi)=0$. Then $\varphi$ is harmonic if and only if $f$ is harmonic and $\sigma$ is parallel.

Proof. A direct computation shows that

$$
\nabla^{*} \nabla \varphi=\left(D^{*} D f\right) \otimes \sigma-\sum_{j}\left(D_{e_{j}} f\right) \otimes\left(\nabla_{e_{j}} \sigma\right)+f \otimes\left(\nabla^{*} \nabla \sigma\right) .
$$

The term $\mathfrak{R}^{\Gamma}(\varphi)$ in Theorem (3.10) vanishes. Thus, since $\varphi$ is harmonic, we have that

$$
\nabla^{*} \nabla \varphi=-\varphi \circ(\operatorname{Ric} \wedge I+2 R) .
$$

Taking the derivative of the condition $\|\sigma\|^{2} \equiv 1$, we find that $\langle\nabla \sigma, \sigma\rangle \equiv 0$. Consequently,

$$
\left\langle\nabla^{*} \nabla \sigma, \sigma\right\rangle \equiv-\sum_{j}\left\langle\nabla_{e_{j}, e_{j}}^{2} \sigma, \sigma\right\rangle \equiv \sum_{j}\left\langle\nabla_{e_{j}} \sigma, \nabla_{e_{j}} \sigma\right\rangle \equiv\|\nabla \sigma\|^{2}
$$

From (3.16) and (3.17) we then conclude that

$$
\begin{aligned}
\left\langle\nabla^{*} \nabla \varphi, \varphi\right\rangle & =\left\langle D^{*} D f, f\right\rangle+f^{2}\|\nabla \sigma\|^{2} \\
& =-\langle f \circ(\operatorname{Ric} \wedge I+2 R), f\rangle .
\end{aligned}
$$

The Bochner-Weitzenböck formula (3.10) applied to scalar-valued 2-forms (let $\mathfrak{g}_{E}$ be trivial), states that $\Delta^{D} f=D^{*} D f+f \circ(\operatorname{Ric} \wedge I+2 R)$. Therefore, (3.18) can be rewritten as:

$$
\left\langle\Delta^{D} f, f\right\rangle+f^{2}\|\nabla \sigma\|^{2}=0 .
$$

Since $\Delta^{D} \geqq 0$ on $M$ we conclude that $\Delta^{D} f=0$ and that $\nabla \sigma=0$ away from the zeros of $f$.

\section{Some Algebraic Facts Specific to Dimension 4}

When $\operatorname{dim} M=4$, the Hodge Star operator * is an involution of $\Lambda^{2} T^{*} M$, giving rise to the decomposition

$$
\Lambda^{2} T^{*} M=\Lambda^{+} T^{*} M \oplus \Lambda^{-} T^{*} M
$$

into eigenspaces for the eigenvalues \pm 1 . Hence, for any vector bundle $F$ over $M$, every $\varphi$ in $\Omega^{2}(F)$ can be written uniquely as $\varphi=\varphi^{+}+\varphi^{-}$where

$$
\varphi^{ \pm}=\frac{1}{2}\left(\mathrm{Id} \pm^{*}\right) \varphi
$$


and

$$
* \varphi^{ \pm}= \pm \varphi^{ \pm}
$$

If $\varphi=\varphi^{+}$, then $\varphi$ is called self-dual.

If $\varphi=\varphi^{-}$, it is called anti-self-dual.

From (2.27) and (4.2), we have the following.

(4.3) Lemma. On a compact oriented 4-manifold $M$, a vector-valued differential 2-form $\varphi$ is harmonic if and only if both $\varphi^{+}$and $\varphi^{-}$are harmonic.

Any vector bundle $E$ over a compact oriented 4-manifold $M$ has associated with it a number $p_{1}(E)$ called the Pontryagin number of $E$. This number is a topological invariant of $E$ and can be computed by the formula

$$
p_{1}(E)=\frac{1}{4 \pi^{2}} \int_{M}\left\langle R^{\nabla} \wedge R^{\nabla}\right\rangle
$$

where $R^{\nabla}$ is the curvature of any connection $\nabla$ on $E$. Writing $R^{\nabla}=R^{\nabla^{+}}+R^{\nabla^{-}}$, we have

$$
\left\langle R^{\nabla} \wedge R^{\nabla}\right\rangle=\left\langle R^{\nabla} \wedge * * R^{\nabla}\right\rangle=\left\langle R^{\nabla} \wedge\left({ }^{*} R^{\nabla+}-{ }^{*} R^{\nabla-}\right)\right\rangle=\left\|R^{\nabla+}\right\|^{2}-\left\|R^{\nabla-}\right\|^{2} .
$$

Of course $\left\|R^{\nabla}\right\|^{2}=\left\|R^{\nabla^{+}}\right\|^{2}+\left\|R^{\nabla-}\right\|^{2}$. Consequently, we have the fundamental inequality

$$
4 \pi^{2}\left|p_{1}(E)\right| \leqq \mathscr{Y} \mathscr{M}(\nabla)
$$

with equality attained if and only if $R^{\nabla}$ is either self-dual or anti-self-dual. In particular, the self-dual (or anti-self-dual) curvatures realize an absolute minimum of the Yang-Mills functional. Such curvatures are, of course, Yang-Mills fields.

All these facts are by now quite standard.

Further topological restrictions of this type for $\mathrm{SO}_{4}$-bundles will be presented in Sect. 8.

There is another algebraic fact which seems less well known, but will be at the heart of our proof of the stability Theorem $\mathrm{B}$ : the $\mathrm{SO}_{4}$-bundles $\Lambda^{+} T M$ and $\Lambda^{-} T M$ are both irreducible and their tensor product is isomorphic to the $\mathrm{SO}_{4}$-bundle $S_{0}^{2} T M$ of traceless symmetric 2-tensors. We will use it in the following form (which after a moment's thought is equivalent to the preceeding statement).

(4.6) Lemma. Let $\varphi^{+}$and $\varphi^{-}$be respectively a self-dual and an anti-self-dual (vector-valued) 2-form on $\mathbb{R}^{4}$. Then for vectors $X, Y \in \mathbb{R}^{4}$, the quantity $\sum_{j=1}^{4} \varphi_{e_{j}, X}^{+} \otimes \varphi_{e_{j}, Y}^{-}$is symmetric in $X$ and $Y$. For ordinary forms, the resulting map from $\Lambda^{+} \mathbb{R}^{4} \otimes \Lambda^{-} \mathbb{R}^{4} \cong \operatorname{Hom}\left(\Lambda^{+} \mathbb{R}^{4}, \Lambda^{-} \mathbb{R}^{4}\right)$ to $S_{0}^{2} \mathbb{R}^{4} \equiv\left\{h \in \operatorname{Hom}\left(\mathbb{R}^{4}, \mathbb{R}^{4}\right): h\right.$ is traceless and symmetric $\}$ is an $\mathrm{SO}_{4}$-isomorphism of $\mathrm{SO}_{4}$-modules. Its inverse is given by $h \mapsto \frac{1}{2} h \wedge I$ where, after restriction, the homomorphism $h \wedge I$ gives a linear map from $\Lambda^{+} \mathbb{R}^{4}$ to $\Lambda^{-} \mathbb{R}^{4}$. 
Proof. We can suppose that $X$ and $Y$ are unit vectors orthogonal to each other. We then pick the orthonormal basis $\left(e_{i}\right)$ such that $e_{1}=X, e_{2}=Y$. Then

$$
\sum_{j=1}^{4} \varphi_{e_{j}, e_{1}}^{+} \otimes \varphi_{e_{j}, e_{2}}^{-}=\varphi_{e_{3}, e_{1}}^{+} \otimes \varphi_{e_{3}, e_{2}}^{-}+\varphi_{e_{4}, e_{1}}^{+} \otimes \varphi_{e_{4}, e_{2}}^{-} .
$$

From the equalities analogous to $\varphi_{e_{3}, e_{1}}^{+}=\varphi_{e_{2}, e_{4}}^{+}, \varphi_{e_{3}, e_{2}}^{-}=\varphi_{e_{1}, e_{4}}^{-}$we get the announced symmetry. The map considered is obviously an $\mathrm{SO}_{4}$-map. Since its image is non-trivial and $S_{0}^{2} \mathbb{R}^{4}$ irreducible, the map is surjective. Since both spaces have dimension 9, it must be an isomorphism. One then checks that the inverse is indeed given by the extension to 2 -forms of a symmetric transformation.

This fact has the following consequences for the riemannian curvature. Viewed as an operator on 2-forms, the curvature splits into three irreducible components under the action of $O_{n}(n \geqq 4)$ : the Weyl conformal curvature tensor $W$, the Ricci traceless part (which is $\frac{1}{n-2} \operatorname{Ric}_{0} \wedge I$ if $\mathrm{Ric}_{0}$ is the traceless part of Ric) and the constant curvature part. In dimension 4 , the Ricci traceless part anticommutes with the Hodge Star-operator *; the other parts commute with *. This fact allows us to identify again the traceless symmetric 2-tensors with linear maps from $\Lambda^{+} T M$ into $\Lambda^{-} T M$, giving another viewpoint on Lemma (4.6).

In fact, more is taking place: there is a further decomposition under the group $\mathrm{SO}_{4}$. The Weyl part $W$ splits into $W^{+}+W^{-}$(where $W^{ \pm}$operates trivially on $\left.\Lambda^{\mp} T M\right)$. This gives rise to a special family of Riemannian 4-manifolds the halfconformally flat spaces. Among them we find some complex manifolds such as $\mathbb{C} P^{2}$ with its canonical metric for which $W^{-}=0$, or the K3-surfaces with their Ricci-flat metrics for which $W^{+}=0$. (For more on these spaces, see $[3,5]$.) As a consequence in the Weitzenböck formula for anti-self-dual 2-forms on $\mathbb{C} P^{2}$, the contribution of the curvature of the base is diagonal as on the standard sphere. This contribution even drops out on self-dual 2 -forms on a Ricci-flat K3 surface.

\section{Isolation Theorems}

In this section we prove a series of isolation results for Yang-Mills fields. The basic idea is to exploit the non-linearity of the Bochner-Weitzenböck formula (3.14). For $\varphi=R^{\nabla}$, this formula implies that

$$
\left\langle\nabla^{*} \nabla R^{\nabla}, R^{\nabla}\right\rangle=-\left\langle R^{\nabla} \circ(\operatorname{Ric} \wedge I+2 R), R^{\nabla}\right\rangle-\varrho\left(R^{\triangleright}\right),
$$

where

$$
\varrho\left(R^{\bigvee}\right) \equiv \sum_{i, j, k=1}^{n}\left\langle\left[R_{e_{i}, e_{j}}^{\nabla}, R_{e_{j}, e_{k}}^{\nabla}\right], R_{e_{k}, e_{i}}^{\nabla}\right\rangle .
$$

When $n=4$, the analogous formulas hold with $R^{\nabla}$ everywhere replaced by $R^{\nabla+}$ which we abbreviate $R^{+}$. Recall that $R^{+}$is harmonic and so formula (3.14) applies [see Lemma (4.3)]. We then examine the last term $\mathfrak{R}\left(R^{+}\right)$of this formula. Writing $R^{\nabla}=R^{+}+R^{-}$gives a splitting of this into two terms, the second of which is of the form

$$
\mathfrak{R}^{-}\left(R^{+}\right)_{X, Y} \equiv \sum_{j=1}^{n}\left\{\left[R_{e_{j}, X}^{-}, R_{e_{j}, Y}^{+}\right]-\left[R_{e_{j}, Y}^{-}, R_{e_{j}, X}^{+}\right]\right\}
$$


By Lemma (4.6), this expression vanishes. We conclude that $\left\langle\mathfrak{H}\left(R^{+}\right), R^{+}\right\rangle=\varrho\left(R^{+}\right)$ and so formula (5.1) holds for $R^{+}$as claimed. Of course, by the same argument (or by a reversal of orientation) we see that this formula also holds for $R^{-}$.

In Sect. 8 we show that when $n=4$ and $G=\mathrm{SO}_{4}$, there is a further decomposition of the curvature, $R^{\nabla}=R_{+}^{+}+R_{-}^{+}+R_{+}^{-}+R_{-}^{-}$, into harmonic components. Since $\left[R_{+}^{\cdot}, R_{-}^{\cdot}\right]=0$ and since $R^{+}=R_{+}^{+}+R_{-}^{+}$, we see easily that $\left\langle\Re\left(R_{+}^{+}\right), R_{+}^{+}\right\rangle$ $=\varrho\left(R_{+}^{+}\right)$. It follows immediately that $R_{+}^{+}$(also $R_{-}^{+}$, etc.) satisfies (5.1).

Now we examine the term $\varrho$ given by (5.2). For any Lie algebra $g$ with a fixed invariant inner product $\langle\cdot, \cdot\rangle$, we have the associated fundamental 3 -form $\Phi_{\mathfrak{g}}$ given by

$$
\Phi_{\mathrm{g}}(U, V, W) \equiv\langle[U, V], W\rangle
$$

for $U, V, W \in \mathfrak{g}$. Now there is a canonical isometry $\Lambda^{2} T M \cong \mathfrak{s o}_{M}$ given by (2.29) and so we may consider $R$ as a linear map

$$
R: \mathfrak{s o}_{M} \rightarrow \mathfrak{g}_{E} .
$$

In $\mathfrak{s o}_{M}$ we have the identities:

$$
\left[e_{i} \wedge e_{j}, e_{k} \wedge e_{l}\right]=\delta_{i l} e_{k} \wedge e_{j}+\delta_{j l} e_{i} \wedge e_{k}+\delta_{i k} e_{j} \wedge e_{l}+\delta_{j k} e_{l} \wedge e_{i}
$$

for all $i, j, k, l$. Hence, we may rewrite (5.2) as

$$
\begin{aligned}
\varrho\left(R^{\bigvee}\right) & =\sum_{i, j, k=1}^{n} \Phi_{\mathfrak{g}^{E}}\left(R_{e_{i}, e_{j}}^{\nabla}, R_{e_{j}, e_{k}}^{\nabla}, R_{e_{k}, e_{i}}^{\nabla}\right) \\
& =\sum_{i, j, k=1}^{n}\left(R^{\nabla *} \Phi_{\mathfrak{g}_{E}}\right)\left(e_{i} \wedge e_{j}, e_{j} \wedge e_{k}, e_{k} \wedge e_{i}\right) \\
& =\left(R^{\nabla *} \Phi_{\mathfrak{g}_{E}}, \Phi_{\mathfrak{s o}_{M}}\right),
\end{aligned}
$$

where, for notational convenience, we define the inner product in $\Lambda^{3} \mathfrak{s o}_{M}^{*}$ by

$$
(\Phi, \Psi)=\sum_{\alpha, \beta, \gamma} \Phi(\alpha, \beta, \gamma) \psi(\alpha, \beta, \gamma)
$$

where $\alpha, \beta$, and $\gamma$ each run over an orthonormal basis of $\mathfrak{s o}_{M}$. result.

Combining (5.1) and (5.4) and integrating by parts gives the following basic

(5.5) Theorem. Let $R^{\nabla}$ be a Yang-Mills field and let $\lambda$ be the minimal eigenvalue of the operator Ric $\wedge I+2 R$ on 2-forms over a compact riemannian manifold $M$. Then

$$
\int_{M}\left\|\nabla R^{\nabla}\right\|^{2} \leqq-\int_{M}\left\{\lambda\left\|R^{\nabla}\right\|^{2}+\left(R^{\nabla *} \Phi_{\mathfrak{g}_{E}}, \Phi_{\mathfrak{s o}_{M}}\right)\right\} .
$$

When $n=4$, this formula holds with $R^{\nabla}$ replaced by $R^{\nabla+}$ and with $\lambda$ replaced by $\lambda^{+}$, the minimal eigenvalue of $\operatorname{Ric} \wedge I+2 R$ on $\Lambda^{+} T M$. (The corresponding statement holds with +'s replaced by -'s.) If, in addition, $G=\mathrm{SO}_{4}$, the formula also holds with $R$ replaced by $R_{+}^{+}, R_{-}^{+}$, etc.

We now observe that the term $\varrho\left(R^{V}\right)=\left(R^{\nabla *} \Phi_{\mathfrak{g}_{E}}, \Phi_{\mathfrak{s v}_{M}}\right)$ is a homogeneous cubic function of $R^{\nabla}$, whereas $\left\|R^{\nabla}\right\|^{2}$ is homogeneous quadratic. For $R^{\nabla}$ sufficiently small 
and $\lambda>0$, this quadratic term will dominate the right hand side of formula (5.5) causing it to become negative. (This, of course, is not possible.) The point at which $\left\|R^{\nabla}\right\|^{2}$ dominates the expression can be estimated concretely, and in some cases, quite accurately. To do this we want to estimate $\left(L^{*} \Phi_{\mathfrak{g}}, \Phi_{\mathbf{s o}_{n}}\right)$ in terms of $\|L\|^{2}$ where $L: \mathfrak{s o}_{n} \rightarrow \mathfrak{g}$ is a linear map and where $\mathfrak{g}$ is any Lie sub-algebra of $\mathfrak{s o}_{N}$. Recall that the inner product on $\mathfrak{g}$ is induced from the canonical one on $\mathfrak{s o}_{N}$ [defined by (2.14)]. Consequently $L^{*} \Phi_{\mathfrak{g}}=L^{*} \Phi_{\mathfrak{s i n}_{N}}$ and for the moment we can ignore $\mathfrak{g}$.

(5.6) Proposition. Let $L: \mathfrak{s o}_{n} \rightarrow \mathfrak{s 0 _ { N }}$ be any linear map. If $\|L\|^{2} \leqq \frac{1}{2}\left(\begin{array}{l}n \\ 2\end{array}\right)$, then

$$
\left(L^{*} \Phi_{\mathfrak{s o}_{N}}, \Phi_{\mathfrak{s o}_{n}}\right) \leqq 2(n-2)\|L\|^{2} .
$$

If $n \geqq 5$, this inequality is strict.

When $n=4$ (respectively $n=3$ ), equality holds if and only if there is an orthogonal splitting $\mathbb{R}^{N}=S_{0} \oplus S_{1}\left(\operatorname{dim} S_{1}=4\right)$ with respect to which $L=0 \oplus \sigma$ where $\sigma$ is one of the two irreducible spin representations of $\mathfrak{s o}_{4}$ (respectively where $\sigma$ is the irreducible spin representation of $\mathfrak{s o}_{3}$ ).

When $n=N=3$, the inequality holds for $\|L\|^{2} \leqq\left(\begin{array}{l}n \\ 2\end{array}\right)=3$. In this case equality is attained if and only if $L: \mathfrak{s o}_{3} \rightarrow \mathfrak{s o}_{3}$ is a Lie algebra isomorphism.

Proof. Note that it suffices to prove the inequality for $\|L\|^{2}=\frac{1}{2}\left(\begin{array}{l}n \\ 2\end{array}\right)$, since for $0 \leqq t \leqq 1$ we then have that

$$
\begin{aligned}
2(n-2)\|t L\|^{2} & =2(n-2) t^{2}\|L\|^{2} \geqq t^{2}\left|\left(L^{*} \Phi_{\mathfrak{s o}_{N}}, \Phi_{\mathfrak{s o}_{n}}\right)\right| \geqq t^{3}\left|\left(L^{*} \Phi_{\mathfrak{s o}_{N}}, \Phi_{\mathfrak{s o}_{n}}\right)\right| \\
& =\left|\left((t L)^{*} \Phi_{\mathfrak{s o}_{N}}, \Phi_{\mathfrak{s v}_{n}}\right)\right| \geqq\left((t L)^{*} \Phi_{\mathfrak{s o}_{N}}, \Phi_{\mathfrak{s o}_{n}}\right) .
\end{aligned}
$$

To compute we let $\left\{e_{i} \wedge e_{j}\right\}_{i<j}$ denote the standard orthonormal basis of $\mathfrak{s o}_{n} \cong \Lambda^{2} \mathbb{R}^{n}$. Then

$$
\|L\|^{2}=\sum_{i<j}\left\|L\left(e_{i} \wedge e_{j}\right)\right\|^{2}
$$

and

$$
\left(L^{*} \Phi_{\mathfrak{s o}_{N}}, \Phi_{\mathfrak{s o}_{n}}\right)=\sum_{i, j, k=1}^{n}\left\langle\left[L\left(e_{i} \wedge e_{j}\right), L\left(e_{j} \wedge e_{k}\right)\right], L\left(e_{k} \wedge e_{i}\right)\right\rangle .
$$

We now introduce the $(n \times n)$-symmetric matrix $\sigma=\left(\left(s_{i j}\right)\right)$ with non-negative entries $s_{i j} \equiv \sqrt{2}\left\|L\left(e_{i} \wedge e_{j}\right)\right\|$. By assumption

$$
\operatorname{trace}\left(\sigma^{2}\right)=\sum_{i, j=1}^{n} s_{i j}^{2}=4\|L\|^{2}=n(n-1) .
$$

We claim that it suffices to prove that

$$
\operatorname{trace}\left(\sigma^{3}\right)=\sum_{i, j, k=1}^{n} s_{i j} s_{j k} s_{k i} \leqq n(n-1)(n-2)
$$


with equality if and only if $s_{i j}=1-\delta_{i j}$. Indeed if this is so, then by Lemma (2.30) we have that

$$
\begin{aligned}
\left(L^{*} \Phi_{\mathfrak{s o}_{N}}, \Phi_{\mathfrak{s o}_{n}}\right) & \leqq \sum_{i, j, k=1}^{n}\left|\left\langle\left[L\left(e_{i} \wedge e_{j}\right), L\left(e_{j} \wedge e_{k}\right)\right], L\left(e_{k} \wedge e_{i}\right)\right\rangle\right| \\
& \leqq \sum_{i, j, k=1}^{n}\left\|\left[L\left(e_{i} \wedge e_{j}\right), L\left(e_{j} \wedge e_{k}\right)\right]\right\|\left\|L\left(e_{k} \wedge e_{i}\right)\right\| \\
& \leqq \sum_{i, j, k=1}^{n} \sqrt{2}\left\|L\left(e_{i} \wedge e_{j}\right)\right\|\left\|L\left(e_{j} \wedge e_{k}\right)\right\|\left\|L\left(e_{k} \wedge e_{i}\right)\right\| \\
& =\frac{1}{2} \sum_{i, j, k=1}^{n} s_{i j} s_{j k} s_{k i} \leqq 2(n-2)\|L\|^{2} .
\end{aligned}
$$

Suppose now that we have equality in each line of (5.7). From the last line we get that $s_{i j}=1-\delta_{i j}$, and so $\left\|L\left(e_{i} \wedge e_{j}\right)\right\|=1 / \sqrt{2}$ for $i \neq j$. From the first and second lines we conclude that, when $i, j, k$ are distinct,

$$
\left[L\left(e_{i} \wedge e_{j}\right), L\left(e_{j} \wedge e_{k}\right)\right]=t_{i j k} L\left(e_{k} \wedge e_{i}\right)
$$

where $t_{i j k}>0$. Taking the inner product with $L\left(e_{k} \wedge e_{i}\right)$ and using the (term by term) equality in (5.7) we see that $t_{i j k}=s_{i j} s_{j k} s_{i k}=1$. Hence, we have

$$
\left[L\left(e_{i} \wedge e_{j}\right), L\left(e_{j} \wedge e_{k}\right)\right]=L\left(e_{k} \wedge e_{i}\right) \text { for all } i, j, k \text { distinct. }
$$

This equation has a number of consequences. For example,

$$
\left\langle L\left(e_{i} \wedge e_{j}\right), L\left(e_{j} \wedge e_{k}\right)\right\rangle=0 \text { for all } i, j, k
$$

since, setting $L_{i j}=L\left(e_{i} \wedge e_{j}\right)$, we have that

$$
\left\langle L_{i j}, L_{j k}\right\rangle=\left\langle\left[L_{j k}, L_{k i}\right], L_{j k}\right\rangle=-\left\langle L_{k i},\left[L_{j k}, L_{j k}\right]\right\rangle=0 .
$$

More generally,

$$
\left\langle L\left(e_{i} \wedge e_{j}\right), L\left(e_{k} \wedge e_{l}\right)\right\rangle=-\left\langle L\left(e_{k} \wedge e_{j}\right), L\left(e_{i} \wedge e_{l}\right)\right\rangle \text { for all } i, j, k, l
$$

since

$$
\left\langle L_{i j}, L_{k l}\right\rangle=\left\langle\left[L_{j k}, L_{k i}\right], L_{k l}\right\rangle=\left\langle L_{j k},\left[L_{k i}, L_{k l}\right]\right\rangle=-\left\langle L_{k j}, L_{i l}\right\rangle .
$$

Another consequence is that setting $\alpha_{i j k l}=\left[L_{i j}, L_{k l}\right]$, we have

$$
\alpha_{i j k l}+\alpha_{i l j k}+\alpha_{i k l j}=0 \text { for all } i, j, k, l \text { distinct. }
$$

To see this, note that

$$
\begin{aligned}
{\left[L_{i j}, L_{k l}\right] } & =\left[L_{i j},\left[L_{l i}, L_{i k}\right]\right]=\left[L_{l i},\left[L_{i j}, L_{i k}\right]\right]+\left[L_{i k},\left[L_{l i}, L_{i j}\right]\right] \\
& =\left[L_{l i}, L_{j k}\right]+\left[L_{i k}, L_{j l}\right] .
\end{aligned}
$$

We now see that $\alpha_{i j k l}$ has the first two symmetries of a Riemann curvature tensor. A standard argument proves that it therefore has the third symmetry, i.e., $\alpha_{i j k l}=\alpha_{k l i j}$. However, from the definition we see that $\alpha_{i j k l}=-\alpha_{k l i j}$, and so we conclude that

$$
\left[L\left(e_{i} \wedge e_{j}\right), L\left(e_{k} \wedge e_{l}\right)\right]=0 \text { for } i, j, k, l \text { distinct }
$$


Comparing (5.8) and (5.12) with (5.3) we conclude that $L: \mathfrak{s o}_{n} \rightarrow \mathfrak{s o}_{N}$ is a Lie algebra homomorphism.

Finally, we observe that by Lemma (2.30) each pair $\left(L\left(e_{i} \wedge e_{j}\right), L\left(e_{j} \wedge e_{k}\right)\right)$, for $i, j, k$ distinct, is conjugate to a pair of Pauli matrices. In particular, each of the endomorphisms $L\left(e_{i} \wedge e_{j}\right)$ is supported in the same 4-dimensional subspace, and by (5.8) each triple $\left(L\left(e_{i} \wedge e_{j}\right), L\left(e_{j} \wedge e_{k}\right), L\left(e_{k} \wedge e_{i}\right)\right)$ for $i, j, k$ distinct is orthogonally equivalent to the standard orthonormal basis of Pauli matrices. The conclusion for $n=3$ and 4 now follows easily.

For $n \geqq 5$ the situation described above is impossible since there exist no nontrivial homomorphisms $\mathfrak{s o}_{n} \rightarrow \mathfrak{s o}_{4}$ for $n \geqq 5 \mathfrak{s o}_{n}$ being simple. To see this directly we note that if $n \geqq 5$ and if $i, j, k, l$ are distinct, then

$$
\left\langle L\left(e_{i} \wedge e_{j}\right), L\left(e_{k} \wedge e_{l}\right)\right\rangle=0 .
$$

This is proved by choosing $m$ distinct from $i, j, k, l$ and using (5.11) and (5.10) as follows :

$$
\begin{aligned}
0 & =\left\langle\left[L_{i j}, L_{m l}\right], L_{m k}\right\rangle+\left\langle\left[L_{i l}, L_{j m}\right], L_{m k}\right\rangle+\left\langle\left[L_{i m}, L_{l j}\right], L_{m k}\right\rangle \\
& =\left\langle L_{i j},\left[L_{m l}, L_{m k}\right]\right\rangle+\left\langle L_{i l},\left[L_{j m}, L_{m k}\right]\right\rangle+\left\langle L_{l j},\left[L_{m k}, L_{i m}\right]\right\rangle \\
& =\left\langle L_{i j}, L_{l k}\right\rangle+\left\langle L_{i l}, L_{k j}\right\rangle+\left\langle L_{l j}, L_{i k}\right\rangle \\
& =-\left\langle L_{i k}, L_{l j}\right\rangle+\left\langle L_{i l}, L_{k j}\right\rangle+\left\langle L_{l j}, L_{i k}\right\rangle \\
& =\left\langle L_{i l}, L_{k j}\right\rangle=-\left\langle L_{i j}, L_{k l}\right\rangle .
\end{aligned}
$$

It follows that if $n \geqq 5$, the matrices $\left\{\sqrt{2} L_{i j}\right\}_{i<j}$ are orthonormal. In particular, $L$ is injective and $L: \mathfrak{s o}_{n} \rightarrow \mathfrak{s o}_{4}$ is impossible.

In the case where $n=N=3$, we can improve (5.7) by using the refinement of Lemma (2.30) given in (2.31). The conclusion in this case is easily obtained.

To complete our proof it remains only to establish the following.

(5.14) Lemma. Let $\sigma=\left(\left(s_{i j}\right)\right)$ be a symmetric $n \times n$ matrix with $s_{i j} \geqq 0$ and $s_{i i}=0$. If $\operatorname{trace}\left(\sigma^{2}\right)=n(n-1)$, then

$$
\operatorname{trace}\left(\sigma^{3}\right) \leqq n(n-1)(n-2)
$$

with equality if and only if $s_{i j}=1-\delta_{i j}$.

Proof. To find the maximum of the function $F(\sigma)=\operatorname{trace}\left(\sigma^{3}\right)$ on the space of symmetric matrices subject to the constraints : $\operatorname{trace}\left(\sigma^{2}\right)=n(n-1), s_{i i}=0$ and $s_{i j} \geqq 0$ we look for all critical points. By the usual Lagrange multiplier technique the matrix $\sigma$ is critical for $F$ if there exist real numbers, $\lambda, \lambda_{1}(1 \leqq i \leqq n)$, and $\lambda_{i j}$ $(1 \leqq i<j \leqq n)$ such that

$$
\sigma^{2}=\lambda \sigma+\sum_{i} \lambda_{i} e_{i}^{*} \otimes e_{i}+\sum_{i<j} \lambda_{i j}\left(e_{i}^{*} \otimes e_{j}+e_{j}^{*} \otimes e_{i}\right),
$$

where $\lambda_{i j} \neq 0$ only if $s_{i j}=0$. (This is the boundary effect.) We conclude that $F(\sigma)$ $=\lambda \operatorname{trace}\left(\sigma^{2}\right)=\lambda n(n-1)$.

Our objective is therefore to show that $\lambda$ is bounded by $n-2$.

We first note that the multipliers $\lambda_{i j}$ are unnecessary when one is interested in a maximum. Indeed suppose $\sigma$ is critical and lies on the boundary $\left\{s_{i j}=0\right\}$ of our 
space of matrices. Then the matrices $\sigma_{t}$ defined for $t \geqq 0$ by

$$
\left[1+\frac{2 t^{2}}{n(n-1)}\right]^{1 / 2} \sigma_{t}=\sigma+t\left(e_{i}^{*} \otimes e_{j}+e_{j}^{*} \otimes e_{i}\right)
$$

are non-negative and satisfy the constraints. Moreover, we have

$$
\left[1+\frac{2 t^{2}}{n(n-1)}\right]^{3 / 2} F\left(\sigma_{t}\right)=F(\sigma)+6 t \sum_{k=1}^{n} s_{j k} S_{k i} .
$$

Note that by (5.15), we have $\lambda_{i j}=\left(\sigma^{2}\right)_{i j}=\sum_{k} s_{i k} s_{i j}$. Assuming $\lambda_{i j}>0$, we see that $F\left(\sigma_{t}\right)$ $>F(\sigma)$ for all $t$ sufficiently small. Consequently, if $F(\sigma)$ is a maximum, all $\lambda_{i j}$ must vanish.

By a permutation of indices we can reduce $\sigma$ to a block matrix $\sigma=\sigma_{1} \oplus \ldots \oplus \sigma_{k}$ where each block $\sigma_{j}$ of size $n_{j}$ cannot be further reduced in this fashion (see [11, Chap. III], for example). It is clear that each block satisfies the equation

$$
\sigma_{j}^{2}=\lambda \sigma_{j}+\Delta_{j}
$$

where $\Delta_{j}$ is a diagonal $n_{j} \times n_{j}$ matrix. Notice then that $\Delta_{j}$ is in fact a scalar matrix $\mu_{j} I$ since $\Delta_{j}$ and $\sigma_{j}$ commute and $\sigma_{j}$ is irreducible. Taking the trace of (5.16) we get that $\mu_{j}=\left(1 / n_{j}\right)$ trace $\sigma_{j}^{2}$ (since trace $\left.\sigma_{j}=0\right)$. On the other hand, since $\sigma_{j}$ is a solution of a polynomial equation of degree 2 with scalar coefficients, $\sigma_{j}$ has at most two eigenvalues, say $\alpha_{j}$ and $\beta_{j}$, which necessarily have opposite signs. We suppose $\alpha_{j}<0$.

By the Perron-Frobenius theorem (cf. [11, p. 53]) the positive eigenvalue of $\sigma_{j}$ must be simple. Therefore, $\beta_{j}=\left(n_{j}-1\right) \alpha_{j}$, and $\lambda=\alpha_{j}+\beta_{j}=-\left(n_{j}-2\right) \alpha_{j}$, and trace $\sigma_{j}^{2}$ $=n_{j}\left(n_{j}-1\right) \alpha_{j}^{2}$.

Consequently, $n_{j}>2$ (since $\lambda>0$ ) and trace $\sigma_{j}^{2}=\left[n_{j}\left(n_{j}-1\right) /\left(n_{j}-2\right)^{2}\right] \lambda^{2}$. It follows that

$$
n(n-1)=\operatorname{trace} \sigma^{2}=\sum_{j=1}^{k} \operatorname{trace} \sigma_{j}^{2}=\sum_{j=1}^{k} \frac{n_{j}\left(n_{j}-1\right)}{\left(n_{j}-2\right)^{2}} \lambda^{2} \geqq k \lambda^{2} .
$$

From (5.17) it is clear that $\lambda$ is maximal when $k=1$. In this case $\lambda=(n-2)$ as expected.

We have now deduced that if $F(\sigma)$ is maximal, then the minimal polynomial of $\sigma$ is $p(\sigma)=(\sigma-n+1)(\sigma+1)$, and the eigenvalue $(n-1)$ appears with multiplicity one. The matrix $\sigma+I$ has only ones on the main diagonal. Moreover, its rank is one, so that for each $i, j(i \neq j)$ there must exist non-zero real numbers $\beta_{i}, \beta_{j}$ so that

$$
\beta_{i}\left(e_{i}+\sum_{k \neq i} s_{i k} e_{k}\right)+\beta_{j}\left(e_{j}+\sum_{k \neq j} s_{j k} e_{k}\right)=0
$$

Consequently, we have that

$$
\begin{aligned}
& \beta_{i} s_{i j}+\beta_{j}=0 \\
& \beta_{i}+\beta_{j} s_{j i}=0 .
\end{aligned}
$$

Since $s_{i j}=s_{j i} \geqq 0$ we conclude that $s_{i j}=1$ for all $i \neq j$. This completes the proof of Lemma (5.14) and of Proposition (5.6). 
(5.18) Remark. The proof of Proposition (5.6) also shows that if $0<\|L\|^{2}<\frac{1}{2}\left(\begin{array}{l}n \\ 2\end{array}\right)$ then $\left(L^{*} \Phi_{\mathfrak{s o}_{N}}, \Phi_{\mathfrak{s o}_{n}}\right)<2(n-2)\|L\|^{2}$.

Combining Theorem (5.5) and Proposition (5.6) gives the following immediate results.

(5.19) Theorem. Any Yang-Mills field $R$ on $S^{n}, n \geqq 5$, which satisfies the pointwise estimate $\|R\|^{2} \leqq \frac{1}{2}\left(\begin{array}{l}n \\ 2\end{array}\right)$ must vanish identically.

Proof. By Proposition (5.6) the integrand on the right in (5.5) is $\geqq 0$ since $\lambda=2(n-2)$ on $S^{n}$. Hence, this integrand vanishes identically (as does $\nabla R$ ). By Proposition (5.6), this implies $R \equiv 0$.

(5.20) Theorem. Let $R$ be a Yang-Mills field on $S^{4}$. If $R^{+}$satisfies the pointwise condition $\left\|R^{+}\right\|^{2}<3$, then $R^{+} \equiv 0$. The corresponding statement holds for $R^{-}$.

Moreover, if the group of the field is $\mathrm{SO}_{4}$ and if $R_{+}^{+}$satisfies the condition $\left\|R_{+}^{+}\right\|^{2}<3$, then $R_{+}^{+} \equiv 0$. The corresponding statements hold for $R_{-}^{+}, R_{+}^{-}, R_{-}^{-}$(see Sect. 8).

Proof. One argues as in (5.19) with $R$ replaced by $R^{+}, R^{-}, R_{+}^{+}$, etc.

Theorem (5.20) gives an explicit $C^{0}$ neighborhood of the self-dual fields on $S^{4}$ in which no other Yang-Mills fields appear. The self-dual fields are "isolated".

We next examine what happens when equality is allowed in the condition $\|R\|^{2}<3$ on $S^{4}$. To state the result it is nice (although not necessary) to mention the associated bundle $E$.

(5.21) Theorem. Let $(E, G, \nabla, R)$ be a Yang-Mills set-up on $S^{4}$ such that $R$ satisfies the pointwise condition $\|R\|^{2} \leqq 3$. Then either $E$ is flat or $E=E_{0} \oplus S$ where $E_{0}$ is flat and where $S$ is one of the (two) 4-dimensional bundles of tangent spinors with the canonical riemannian connection.

Proof. Arguing as above we see that either $R \equiv 0$ or $\|R\|^{2} \equiv 3$ and $\nabla R \equiv 0$. In the latter case, Proposition (5.6) implies that there is an orthogonal splitting $E=E_{0} \oplus S$ where $E_{0}$ is flat, where $S$ is 4-dimensional, and where $-R: \mathfrak{s o}_{M} \rightarrow \mathfrak{s o}_{S}$ is one of the 2 fundamental spin representations $\sigma^{+}$or $\sigma^{-}$at each point. Let us assume that it is the positive one at each point. (The other case is similar.) Then $R^{-} \equiv 0$ and $-R: \mathfrak{s o}_{M}^{+} \rightarrow \mathfrak{s o}_{S}$ is an isometric bundle injection which, since $\nabla(R)=[\nabla, R]=0$, is connection preserving. Hence,

$$
\mathfrak{s o}_{S}=\mathfrak{s o}_{M}^{+} \oplus \underline{\mathfrak{s}}_{3},
$$

where $\underline{\mathfrak{s}}_{3}$ denotes the flat bundle. (Note that $\underline{\mathfrak{s}}_{3}$ corresponds to a parallel quaternion structure on $S$.) Equation (5.22) implies that $S$, pulled back over the principal $\mathrm{Spin}_{4}$-bundle of $S^{4}$, is canonically trivialized and transforms according to the representation $\sigma^{+}$. This completes the proof.

The same argument can be applied to $R^{-}$. We conclude that if $\left\|R^{-}\right\|^{2} \leqq 3$, then either $R^{-}=0$ or the following is true. There is an orthogonal splitting $E=E_{0} \oplus V$ where $V$ is 4-dimensional, and

$$
-R^{-}: \mathfrak{s o}_{M}^{-} \rightarrow \mathfrak{s o}_{V}^{-} \subset \mathfrak{g}_{E}
$$


is a connection preserving bundle isometry. (Recall that $\operatorname{since} \operatorname{dim} V=4$, there is a canonical splitting $\mathfrak{s o}_{V}=\mathfrak{s o}_{V}^{+} \oplus \mathfrak{s o}_{V}^{-}$.) This means that $E_{0}$ is self-dual and that

$$
\mathfrak{s \mathfrak { o } _ { V }} \cong \mathfrak{s o}_{V}^{+} \oplus \mathfrak{s o}_{M}^{-},
$$

where $\mathfrak{s o}_{V}^{+}$is also self-dual. It follows that the principal $\operatorname{Spin}_{4}$-bundle of $V$ can be written as a Whitney sum

$$
P_{\text {Spin }_{4}}(V)=P_{\mathrm{SU}_{2}} \oplus P_{\mathrm{SU}_{2}}\left(S^{-}\right),
$$

where $S^{-}$is the canonical spin bundle above and where the connection on $P_{\mathrm{SU}_{2}}$ is self-dual. This is one of the connections satisfying the minimum conditions (8.7). From $(8.12)$ we see that $\frac{1}{2} p_{1}(V)-\chi(V)=2$. (The reader should consult Sect. 8 for fuller details.) Summarizing the above gives the following.

(5.24) Theorem. Let $(E, G, \nabla, R)$ be a Yang-Mills set-up on $S^{4}$ and assume that $R$ satisfies the pointwise condition $\left\|R^{-}\right\|^{2} \leqq 3$. Then either $E$ is self-dual or $E=E_{0} \oplus V$ where $E_{0}$ is self-dual and where $V$ is a 4-dimensional bundle satisfying (5.23) and the connection on the first factor is also self-dual.

A corresponding theorem for $R^{+}$is obtained by reversing orientations.

We now consider the 3 -sphere. Here the results are simple to state.

(5.25) Theorem. Let $(E, G, \nabla, R)$ be a Yang-Mills set-up on $S^{3}$. If $\|R\|^{2}<\frac{3}{2}$, then $E$ is flat. If $\|R\|^{2} \leqq \frac{3}{2}$, then either $E$ is flat or $E=E_{0} \oplus S$ where $E_{0}$ is flat and $S$ is the 4-dimensional tangent spin bundle with the riemannian connection.

If $\operatorname{dim}(E)=3$ and $\|R\|^{2}<3$, then either $E$ is flat or $E=T S^{3}$ with the riemannian connection.

Proof. The argument for the first part is entirely similar to the argument for Theorem (5.21). For the second part, we use the last statement in (5.6) to conclude that if $R \neq 0$, then $-R: \mathfrak{s o}_{M} \rightarrow \mathfrak{s o}_{E}$ is a connection preserving bundle isometry. Hence, taking the composition

$$
T M \stackrel{*}{\longrightarrow} \Lambda^{2} T M=\mathfrak{s o}_{M} \stackrel{-R}{\longrightarrow} \mathfrak{s o}_{E}=\Lambda^{2} E \stackrel{*}{\longrightarrow} E
$$

gives an equivalence $T M=E$, and the proof is complete.

In order to be able to apply Theorem (5.5) for general dimensions $n$ we need Ric $\wedge I+2 R$ to be a positive operator. This is insured for example if the curvature operator of $M$ is positive (see [6, p. 74]). In this case it is known that $M$ must be a homology sphere by a nice theorem of Gallot and Meyer [10]. The results we just proved still hold with the uniform bound on $\left\|R^{\nabla}\right\|^{2}$ reduced to

$$
\left\|R^{\nabla}\right\|^{2} \leqq \frac{1}{16} \frac{n(n-1)}{(n-2)^{2}} \lambda^{2}
$$

The 4-dimensional case is more interesting since one can consider separately the positive and the negative 2-forms. In particular we have the following.

(5.26) Theorem. Let $M$ be a compact riemannian 4-manifold which is half conformally flat (say, $W^{-}=0$ ) and has positive scalar curvature $\kappa$. Then any Yang-Mills field $R$ on $M$ which satisfies the pointwise condition

$$
\left\|R^{-}\right\|^{2}<\frac{1}{4} \kappa
$$


is self-dual. (A similar statement with -'s replaced by +'s is obtained by reversal of orientation.)

This theorem covers the case of $\mathbb{C} P^{2}$ with the Fubini-study metric where $\kappa=24$.

It is a theorem of $N$. Hitchin that half conformally flat Einstein manifolds with positive scalar curvature are isometric to either $S^{4}$ or $\mathbb{C} P^{2}$. It is conjectured that this conclusion remains if one supposes the manifold to have non vanishing signature and the scalar curvature to be constant.

\section{The Second Variation}

In this section we establish the second variational formula for the Yang-Mills functional at a critical point, i.e., at a Yang-Mills connection. In fact we shall derive three distinct formulas. The first is completely general. The other two are valid only for infinitesimal deformations of the connection, i.e., for elements $B \in \Omega^{1}\left(\mathfrak{g}_{E}\right) \cong T \mathscr{C}_{E}$ which satisfy the transversality hypothesis $\delta^{\nabla} B=0$ [see (2.15) and (2.16)]. However, in these latter cases the formula involves an elliptic second order operator.

We shall work in the general setting developed in Sect. 2. We suppose that $\nabla^{t}$, $|t|<\varepsilon$, is a smooth family of connections on $E$ where $\nabla=\nabla^{0}$ is Yang-Mills, and we write

$$
\nabla^{t}=\nabla+A^{t}
$$

where $A^{t} \in \Omega^{1}\left(\mathfrak{g}_{E}\right)$ for each $t$. Letting $R^{t}$ denote the curvature of $V^{t}$, we then have

$$
R^{t}=R^{\nabla}+d^{\nabla} A^{t}+\frac{1}{2}\left[A^{t} \wedge A^{t}\right]
$$

[see (2.20)]. The infinitesimal variation of connection associated to $\nabla^{t}$ at $t=0$ is just

$$
\left.B \equiv \frac{d A^{t}}{d t}\right|_{t=0} .
$$

Note that $B \in \Omega^{1}\left(\mathfrak{g}_{E}\right)$, and thereby we have the natural identification: $\Omega^{1}\left(\mathfrak{g}_{E}\right) \cong T_{\nabla} \mathscr{C}$. We have defined an endomorphism $\mathfrak{R}^{\nabla}$ of this space by setting

$$
\boldsymbol{K}^{\nabla}(\varphi)_{X} \equiv \sum_{j=1}^{n}\left[R_{e_{j}, X}^{\nabla}, \varphi_{e_{j}}\right]
$$

for $\varphi \in \Omega^{1}\left(\mathfrak{g}_{E}\right)$, where $\left(e_{1}, \ldots, e_{n}\right)$ is any orthonormal basis of tangent vectors to $M$ at the point in question. Our first version of the formula is as follows.

(6.5) Theorem. Suppose $\nabla=\nabla^{0}$ is a Yang-Mills connection. Then the second variation of the Yang-Mills functional is given by

$$
\left.\frac{d^{2}}{d t^{2}} \mathscr{Y} \mathscr{M}\left(\nabla^{t}\right)\right|_{t=0}=\int_{M}\left\langle\delta^{\nabla} d^{\nabla} B+\mathcal{R}^{\nabla}(B), B\right\rangle,
$$

where $B=\left.\frac{d}{d t} V^{t}\right|_{t=0}$. 
Proof. It follows immediately from (6.2) that: $\left.\frac{1}{2}\left(d^{2} / d t^{2}\right)\left\|R^{t}\right\|^{2}\right|_{t=0}=\left\langle d^{\nabla} B, d^{\nabla} B\right\rangle$ $+\left\langle d^{\nabla} C+[B \wedge B], R^{\triangleright}\right\rangle$ where $C=\left.\left(d^{2} / d t^{2}\right) V^{t}\right|_{t=0}$. Integrating (by parts) and using the condition

$$
\delta^{\nabla} R^{\nabla}=0
$$

we see that the term involving $C$ vanishes. (This was expected since at a critical point the Hessian of a functional is intrinsic and hence depends only on the first order part.)

$$
\begin{aligned}
\left\langle[B \wedge B], R^{\triangleright}\right\rangle & =\sum_{i<j}\left\langle 2\left[B_{e_{t}}, B_{e_{j}}\right], R_{e_{i}, e_{j}}^{\nabla}\right\rangle \\
& =\sum_{i, j}\left\langle B_{e_{i}},\left[B_{e_{j}}, R_{e_{i}, e_{j}}^{\nabla}\right]\right\rangle \\
& =\sum_{i}\left\langle B_{e_{t}}, \mathfrak{R}^{\nabla}(B)_{e_{\imath}}\right\rangle \\
& =\left\langle B, \mathfrak{R}^{\nabla}(B)\right\rangle,
\end{aligned}
$$

and the formula is now obvious.

We now restrict our variations to those whose first order part is transversal to the orbit of the gauge group. The next result follows immediately from Theorem (6.5) and the Bochner-Weitzenböck formula (3.2).

(6.8) Theorem. Suppose $\nabla=\nabla^{0}$ is a Yang-Mills connection and $B=\left.\frac{d}{d t} \nabla^{t}\right|_{t=0}$. Suppose also that $\delta^{\nabla} B=0$. Then

$$
\left.\frac{d^{2}}{d t^{2}} \mathscr{Y} \mathscr{M}\left(\nabla^{t}\right)\right|_{t=0}=\int_{M}\left\langle\mathscr{S}^{\nabla}(B), B\right\rangle,
$$

where

$$
\begin{aligned}
\mathscr{S}^{\nabla}(B) & =\Delta^{\nabla} B+\mathfrak{S}^{\nabla}(B) \\
& =\nabla^{*} \nabla B+B \circ \mathrm{Ric}+2 \mathfrak{S}^{\nabla}(B) .
\end{aligned}
$$

(6.9) Corollary. Let $\nabla$ and $B$ be as in (6.8) and suppose $M$ is an Einstein manifold with $\mathrm{Ric}=k \cdot \mathrm{Id}$. . Then

$$
\left.\frac{d^{2}}{d t^{2}} \mathscr{Y} \mathscr{M}\left(\nabla^{t}\right)\right|_{t=0}=\int_{M}\left\langle\nabla^{*} \nabla B+k B+2 \mathcal{I}^{\nabla}(B), B\right\rangle .
$$

Observe that the operator $\mathscr{S}^{\nabla}$ is elliptic and self-adjoint, and that the operator $\nabla^{*} \nabla$ is $\geqq 0$. It follows that the restriction of $\mathscr{S}^{\nabla}$ to the subspace $T^{0} \equiv \operatorname{ker}\left(\delta^{\nabla}\right) \subset \Omega^{1}\left(\mathfrak{g}_{E}\right)$ has eigenvalues $\lambda_{1}<\lambda_{2}<\ldots \rightarrow \infty$, with associated finite dimensional eigenspaces $E_{\lambda_{1}}, E_{\lambda_{2}}, \ldots$ We can therefore introduce the following concepts from Morse theory (cf. $[13,14]$ ).

(6.10) Definitions. The index of a Yang-Mills connection $\nabla$ is the dimension $i(\nabla)=\operatorname{dim}\left(\underset{\lambda<0}{\bigoplus} E_{\lambda}\right)$. The nullity $\nabla$ is the dimension $n(\nabla)=\operatorname{dim}\left(E_{0}\right)$. 
Certainly one of the major problems in the field is to understand the relationship of these numbers to the topology of the gauge group $\mathscr{G}_{E}$. The YangMills functional descends to a function

$$
\mathscr{Y} \mathscr{M}^{0}: \mathscr{C}_{E} / \mathscr{G}_{E} \rightarrow \mathbb{R}
$$

and, roughly speaking, the space $\mathscr{C}_{E} / \mathscr{G}_{E}$ can be identified with the classifying space $B \mathscr{G}_{E}$. [In some cases, for example that of a non-trivial $\mathrm{SU}_{2}$-bundle over $S^{4}$, we actually have $\mathscr{C}_{E} / \mathscr{G}_{E} \cong B \mathscr{G}_{E}$. However, in general one must worry that the action of $\mathscr{G}_{E}$ on $\mathscr{C}_{E}$ is not free, i.e., that there may exist $\nabla \in \mathscr{C}_{E}$ and $g \in \mathscr{G}_{E}, g \neq 1$, such that $\nabla^{g}=\nabla$. In this case we note that $\nabla \circ g=g \circ \nabla$, i.e., $\nabla(g)=0$, which implies a reduction of the structure group of $E$.] Nevertheless, a naive application of standard Morse theory to the function (6.11) gives direct relationships between the topology of $B \mathscr{G}_{E}$ and the number of critical points of $\mathscr{Y} \mathscr{M}$ with a fixed index. Just recently Uhlenbeck [16] has succeeded in establishing the analytic tools required for an appropriate Morse theory when $\operatorname{dim}(M)=2$ or 3. If the Morse theory can be successfully completed, Atiyah and Bott have shown that one obtains new proofs of a number of profound results in algebraic geometry.

The fields of interest in this paper are those which are local minima of the functional. Thus we introduce the following notion.

(6.12) Definition. A Yang-Mills connection $\nabla$ is said to be stable if $i(\nabla)=n(\nabla)=0$, that is, if

$$
\int_{M}\left\langle\mathscr{S}^{\nabla}(B), B\right\rangle>0
$$

for all non-zero $B \in T_{\nabla}^{0}=\operatorname{ker}\left(\delta^{\square}\right)$.

(6.13) Note that if $\mathscr{S}^{\nabla}>0$ on $\Omega^{1}\left(\mathfrak{g}_{E}\right)$, then $\mathscr{S}^{\nabla}>0$ on the subspace $T_{\nabla}^{0}$, and $\nabla$ is stable. We caution the reader to note, however, that the second variation is given by $\mathscr{S}^{\nabla}$ only on the subspace $T_{\nabla}^{0}$.

(6.14) Note that weak stability (second variation $\geqq 0$ ) is equivalent to the condition $i(\nabla)=0$. Thus, in particular, stability implies weak stability.

\section{The Stability Theorems}

In this section we shall explore the structure of weakly stable Yang-Mills fields on the euclidean $n$-sphere $S^{n}=\left\{x \in \mathbb{R}^{n+1}:\|x\|=1\right\}$. It turns out that on $S^{n}$ there is a finite dimensional family of vector fields which tend to decrease the energy of any Yang-Mills field. Using this family we find strong restrictions on any weakly stable field on $S^{4}$. In particular, when $G=\mathrm{SU}_{2}, \mathrm{SU}_{3}$ or $U_{2}$, we show that any such field is either self-dual or anti-self-dual.

We begin with a general observation. Fix a Yang-Mills connection on a riemannian manifold $M$ and consider a 2-form $\varphi \in \Omega^{2}\left(\mathfrak{g}_{E}\right)$. Then for each tangent vector field $V$ on $M$, the contraction $i_{\mathrm{v}} \varphi \in \Omega^{1}\left(\mathfrak{g}_{E}\right)$ defined by setting

$$
\left(i_{\mathbf{V}} \varphi\right)_{X} \equiv \varphi_{\mathbf{V}, X}
$$


is an infinitesimal variation of the connection. Suppose now that $V$ is of gradient type, i.e., that

$$
\left\langle D_{X} V, Y\right\rangle=\left\langle D_{Y} V, X\right\rangle \text { for all } X, Y,
$$

where $D$ is the Levi-Civita connection on $M$. [Condition (7.2) is equivalent to the fact that the dual 1 -form $\omega(\cdot) \equiv\langle V, \cdot\rangle$ is closed.]

(7.3) Lemma. Let $B=i_{\mathrm{V}} \varphi$ where $\varphi \in \Omega^{2}\left(\mathfrak{g}_{E}\right)$ satisfies $\delta^{\nabla} \varphi=0$ and where $V$ is a vector field of gradient type. Then $\delta^{\nabla} B=0$.

Proof. Fix $x \in M$ and let $\left(e_{1}, \ldots, e_{n}\right)$ be a local orthonormal tangent frame field such that $\left(D e_{j}\right)(x)=0$ for each $j$. Write $D_{e_{i}} V=\sum a_{i j} e_{j}$, and note that $a_{i j}=a_{j i}$ for all $i, j$ by (7.2). Consequently, at $x$ we have

$$
\begin{aligned}
\delta^{\nabla} B & =-\sum_{j}\left(\nabla_{e_{j}} B\right)_{e_{j}}=-\sum_{j} \nabla_{e_{j}}\left(B_{e_{j}}\right) \\
& =-\sum_{j} \nabla_{e_{j}}\left(\varphi_{\mathrm{V}, e_{j}}\right) \\
& =-\sum_{j}\left\{\left(\nabla_{e_{j}} \varphi\right)_{\mathrm{V}, e_{j}}+\varphi_{D_{e_{j}} \mathrm{~V}, e_{j}}\right\} \\
& =-\left(\delta^{\nabla} \varphi\right)_{\mathrm{V}}+\sum_{i, j} a_{i j} \varphi_{e_{l}, e_{j}}=0
\end{aligned}
$$

since $\delta^{\nabla} \varphi=0$ and $\varphi_{e_{i}, e_{j}}$ is skew-symmetric in $i$ and $j$.

We now consider on $S^{n}$ the special, finite-dimensional space of vector fields

$$
\mathscr{V} \equiv\left\{\operatorname{grad} f: f=\left.F\right|_{S^{n}} \text { and } F: \mathbb{R}^{n+1} \rightarrow \mathbb{R} \text { is linear }\right\} .
$$

There is a natural isomorphism $\mathbb{R}^{n+1} \stackrel{\underset{\sim}{\rightarrow}}{\mathscr{V}}$ which associates to each $V \in \mathbb{R}^{n+1}$ the vector field $V$ given by

$$
V(x)=v-\langle v, x\rangle x
$$

for $x \in S^{n}$. Note that $V=\operatorname{grad} f$ where $f(x)=\langle v, x\rangle$.

(7.5) Lemma. Each $V \in \mathscr{V}$ satisfies

(i) $D_{X} V=-f X$,

(ii) $D * D V=V$,

where D denotes the Levi-Civita connection of the standard metric on $S^{n}$ and where $f$ is as above.

Proof. Let $\bar{D}$ denote the riemannian connection on $\mathbb{R}^{n+1}$, and let $(\cdot)^{T}: T_{x} \mathbb{R}^{n+1} \rightarrow T_{x} S^{n}$ denote orthogonal projection. Then it is standard that $D Y=(\bar{D} Y)^{T}$ for any tangent vector field $Y$ on $S^{n}$. It then follows from (7.5) that

$$
D_{X} V=\left[\bar{D}_{X}(v-\langle v, x\rangle x)\right]^{T}=-\langle v, x\rangle X=-f(x) X
$$

since $v$ is parallel in $\mathbb{R}^{n+1}$ and since $x$ is normal to $S^{n}$ at $x$. This proves (i). For (ii) we fix $x \in S^{n}$ and choose a local frame field $\left(e_{1}, \ldots, e_{n}\right)$ near $x$. Then, $D^{*} D V$ $=-\sum\left(D_{e_{j}} D_{e_{j}} V-D_{D_{e_{j}} e_{j}} V\right)=\sum\left\langle v, e_{j}\right\rangle e_{j}=v^{T}=V$, and the proof is complete. 
(7.6) Remark. The vector fields belonging to $\mathscr{V}$ are exactly the gradients of the eigenfunctions of the Laplacian corresponding to the first non-zero eigenvalue on $S^{n}$. It follows immediately from 7.5(i) that these fields are conformal. In fact $\mathscr{V}$ is the orthogonal complement to the Killing vector fields in the space of all conformal vector fields on $S^{n}$.

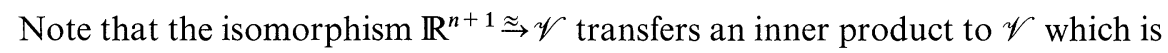
invariant under the natural action of $O_{n+1}$.

We are now in a position to state one of our key results.

(7.7) Theorem. Let $(E, P, G, \nabla)$ be any Yang-Mills set-up over the euclidean sphere $S^{n}$, and suppose $\varphi \in \Omega^{2}\left(\mathfrak{g}_{E}\right)$ is harmonic, i.e., $\delta^{\nabla} \varphi=d^{\nabla} \varphi=0$. Associate to $\varphi$ a quadratic form $Q_{\varphi}$ on $\mathscr{V}$ by setting

$$
Q_{\varphi}(V)=\left.\frac{d^{2}}{d t^{2}} \mathscr{Y} \mathscr{M}\left(\nabla^{t}\right)\right|_{t=0}
$$

where $\nabla^{t}=\nabla+t\left(i_{\mathrm{v}} \varphi\right)$. Then

$$
\operatorname{trace}\left(Q_{\varphi}\right)=2(4-n) \int_{S^{n}}\|\varphi\|^{2} .
$$

Proof. By Lemma (7.3) we know that $\delta^{\nabla}\left(i_{\mathrm{V}} \varphi\right)=0$ for all $V \in \mathscr{V}$ and so we may use formula (6.8) for the second variation. Consequently

$$
\operatorname{trace}\left(Q_{\varphi}\right)=\int_{S^{n}} \operatorname{trace}\left(q_{\varphi}\right),
$$

where $q_{\varphi}(V) \equiv\left\langle\mathscr{S}^{\nabla}\left(i_{\mathrm{v}} \varphi\right), i_{\mathrm{v}} \varphi\right\rangle$. To compute the trace of $q_{\varphi}$ at a point $x \in \mathscr{S}^{n}$ we choose an orthonormal basis $\left(\varepsilon_{0}, \varepsilon_{1}, \ldots, \varepsilon_{n}\right)$ of $\mathscr{V}$ adapted to this point. We let $\varepsilon_{0}, \ldots, \varepsilon_{n}$ correspond, under the isomorphism $\mathscr{V} \cong \mathbb{R}^{n+1}$, to the vectors $x, e_{1}, \ldots, e_{n}$ where $\left(e_{1}, \ldots, e_{n}\right)$ form an orthonormal basis of $T S^{n}$. We see from (7.4) that

$$
\varepsilon_{0}(x)=0, \varepsilon_{1}(x)=e_{1}, \ldots, \varepsilon_{n}(x)=e_{n} .
$$

To apply the formula (6.9) we need to compute $\nabla^{*} \nabla\left(i_{\mathrm{V}} \varphi\right)$ for $V \in \mathscr{V}$. Choose local orthonormal tangent fields $\varepsilon_{1}, \ldots, \varepsilon_{n}$ on $S^{n}$ such that $\left(D \varepsilon_{j}\right)(x)=0$, and let $X=\sum a_{j} \varepsilon_{j}$ be any linear combination of these fields. Then at the point $x$,

$$
\begin{aligned}
{\left[\nabla^{*} \nabla\left(i_{\mathrm{V}} \varphi\right)\right]_{X} } & =-\sum\left[\nabla_{\varepsilon_{j}, \varepsilon_{j}}^{2}\left(i_{\mathrm{V}} \varphi\right)\right]_{X}=-\sum\left[\nabla_{\varepsilon_{j}} \nabla_{\varepsilon_{j}}\left(i_{\mathrm{V}} \varphi\right)\right]_{X} \\
& =-\sum\left\{\nabla_{\varepsilon_{j}}\left[\left(\nabla_{\varepsilon_{j}} i_{\mathrm{V}} \varphi\right)_{X}\right]-\left(\nabla_{\varepsilon_{j}} i_{\mathrm{V}} \varphi\right)_{D_{\varepsilon_{j}} X}\right\} \\
& =-\sum \nabla_{\varepsilon_{j}}\left[\nabla_{\varepsilon_{j}}\left[\left(i_{\mathrm{V}} \varphi\right)_{X}\right]-\left(i_{\mathrm{V}} \varphi\right)_{D_{\varepsilon_{j}} X}\right] \\
& =-\sum \nabla_{\varepsilon_{j}}\left[\nabla_{\varepsilon_{j}}\left(\varphi_{\mathrm{V}, X}\right)-\varphi_{\mathrm{V}, D_{\varepsilon_{j}} X}\right] \\
& =-\sum \nabla_{\varepsilon_{j}}\left[\left(\nabla_{\varepsilon_{j}} \varphi\right)_{\mathrm{V}, X}+\varphi_{D_{\varepsilon_{j}} \mathrm{~V}, X}\right] \\
& =\left(\nabla^{*} \nabla \varphi\right)_{\mathrm{V}, X}-2 \sum\left(\nabla_{\varepsilon_{j}} \varphi\right)_{D_{\varepsilon_{j}} \mathrm{~V}, X}+\varphi_{D^{*} D \mathrm{~V}, X} .
\end{aligned}
$$

Consequently, using (7.5) and the fact that $\delta^{\nabla} \varphi=0$, we conclude that

$$
\left(\nabla^{*} \nabla\left(i_{\mathrm{v}} \varphi\right)\right)_{X}=\left(\nabla^{*} \nabla \varphi\right)_{\mathrm{v}, X}+\varphi_{\mathrm{v}, X} .
$$


Since Ric $=(n-1)$ Id. on $S^{n}$, we have from (6.9) and (7.10) that

$$
\mathscr{S}^{\nabla}\left(i_{\mathrm{V}} \varphi\right)_{.}=\left(\nabla^{*} \nabla \varphi\right)_{\mathrm{V},}+n \varphi_{\mathrm{V}, \cdot}+2 \sum_{i=1}^{n}\left[R_{e_{i},}, \varphi_{\mathrm{V}, e_{i}}\right] .
$$

Since $\varphi$ is harmonic, we have the following Bochner-Weitzenböck formula [see (3.14)]

$$
\left(\nabla^{*} \nabla \varphi\right)_{\mathrm{V}, \cdot}=-2(n-2) \varphi_{\mathrm{V}, \cdot}-\sum_{i=1}^{n}\left\{\left[R_{e_{i}, \mathrm{~V}}^{\nabla}, \varphi_{e_{i},}\right]-\left[R_{e_{i},}^{\nabla}, \varphi_{e_{i}, \mathrm{~V}}\right]\right\} .
$$

Combining these two equations we see that

$$
\mathscr{S}^{\nabla}\left(i_{\mathrm{V}} \varphi\right)=(4-n) i_{\mathrm{V}} \varphi-\sum_{i=1}^{n}\left\{\left[R_{e_{i},}^{\nabla}, \varphi_{e_{i}, \mathrm{~V}}\right]+\left[R_{e_{i}, \mathrm{~V}}^{\nabla}, \varphi_{e_{i},},\right]\right\} .
$$

Therefore, we have at $x$ that

$$
\operatorname{trace}\left(q_{\varphi}\right)=\sum_{j=0}^{n}\left\langle\mathscr{S}^{\nabla}\left(i_{\varepsilon_{j}} \varphi\right), i_{\varepsilon_{j}} \varphi\right\rangle=(4-n) \sum_{j=0}^{n} \sum_{k=1}^{n}\left\langle\varphi_{\varepsilon_{j}, e_{k}}, \varphi_{\varepsilon_{j}, e_{k}}\right\rangle .
$$

Note that the contribution from the second term of (7.11) drops out because we have taken the inner product of a symmetric and a skew-symmetric form.

Using (7.8) we finally obtain at $x$ that

$$
\operatorname{trace}\left(q_{\varphi}\right)=2(4-n) \sum_{j<k}\left\|\varphi_{e_{j}, e_{k}}\right\|^{2}=2(4-n)\|\varphi\|^{2} .
$$

This completes the proof of Theorem (7.7).

Note that in the proof of Theorem (7.7), the introduction of the integrated quadratic form $Q_{\varphi}$ was used only to get an interpretation in terms of the second variation of $\mathscr{Y} \mathscr{M}$. In particular, we insist that in dimension 4 we get the vanishing of the density $q_{\varphi}$.

Since $\nabla$ is a Yang-Mills connection, we can always choose $\varphi=R^{\nabla}$ in Theorem (7.7). Thus we have the following immediate consequence.

(7.11) Theorem (J. Simons). There are no weakly stable Yang-Mills fields on $S^{n}$ for $n \geqq 5$.

Note that when $n=4$, Theorem (7.7) asserts that $\operatorname{trace}\left(Q_{\varphi}\right)=0$. Using this fact we now prove the following.

(7.12) Theorem. Any weakly stable Yang-Mills fields on $S^{4}$ with structure group $\mathrm{SU}_{2}, \mathrm{SU}_{3}$ or $U_{2}$ is either self-dual or anti-self-dual.

Proof. Consider a weakly stable Yang-Mills field with group $G$ on $S^{4}$. Let $\mathscr{I}(B, B)$ be the quadratic form on $\Omega^{1}\left(\mathfrak{g}_{E}\right)$ given by the second variation of the functional at this connection. Since $\mathscr{I}(B, B) \geqq 0$, we see that $\mathscr{I}(B, B)=0$ if and only if $B$ is in the null space of this quadratic form. In particular, if $\mathscr{I}(B, B)=0$ and $\delta^{\nabla} B=0$, then $\mathscr{S}^{\mathbb{P}}(B)=0$. Consequently, Theorem (7.7) implies that if $\varphi \in \Omega^{2}\left(\mathfrak{g}_{E}\right)$ is harmonic and $V \in \mathscr{V}$, then

$$
\mathscr{S}^{\nabla}\left(i_{\mathrm{v}} \varphi\right)=0 .
$$


It follows immediately from Lemma (2.31) that since $R^{\nabla}$ is harmonic, so are $R^{+}=\left(R^{\eta}\right)^{+}$and $R^{-}=\left(R^{\eta}\right)^{-}$. Consequently, by (7.13) we know that

$$
\mathscr{S}^{\nabla}\left(i_{\mathrm{V}} R^{+}\right)=0
$$

for all $V \in \mathscr{V}$. (The corresponding statement is true for $R^{-}$.) Using (7.9) and writing $R^{\nabla}=R^{+}+R^{-}$, we conclude from (7.14) that

$$
\left(\nabla^{*} \nabla R^{+}\right)_{X, Y}+4 R_{X, Y}^{+}+2 \sum_{j=1}^{4}\left[R_{e_{j}, X}^{+}, R_{e_{j}, Y}^{+}\right]=-2 \sum_{j=1}^{4}\left[R_{e_{j}, X}^{+}, R_{e_{j}, Y}^{-}\right]
$$

for all tangent fields $X, Y$ on $S^{4}$. The left hand side of (7.15) is clearly skewsymmetric in $X$ and $Y$. By Lemma (4.6) the right hand side is symmetric in $X$ and $Y$. Consequently, the $\mathfrak{g}_{E}$-valued tensor

$$
\tau_{X, Y} \equiv \sum_{j=1}^{4}\left[R_{e_{j}, X}^{+}, R_{e_{j}, Y}^{-}\right]
$$

vanishes for all $X$ and $Y$, i.e., $\tau=0$.

(7.16) Proposition. At each point $x \in S^{4}$,

$$
\left[R_{X, Y}^{+}, R_{Z, W}^{-}\right]=0
$$

for all $X, Y, Z, W \in T_{x} S^{4}$. Consequently, at each point $x \in S^{4}$,

$$
\left[\mathfrak{a}_{x}^{+}, \mathfrak{a}_{x}^{-}\right]=0,
$$

where $\mathfrak{a}_{x}^{ \pm} C\left(\mathfrak{g}_{E}\right)_{x}$ is the Lie subalgebra generated by the curvature transformations $R_{X, Y}^{ \pm}$for $X, Y \in T_{x} S^{4}$.

Proof. This proposition can be deduced from the second part of Lemma (4.6) as follows. Since $\tau=0, \tau \wedge I=0$. However, one can easily see that

$$
\left\langle\frac{1}{2}(\tau \wedge I)_{X, Y}, Z \wedge W\right\rangle=\left[R_{X, Y}^{+}, R_{Z, W}^{-}\right] .
$$

For the convenience of the reader who is uncomfortable with abstract linear algebra, we offer a second, more elementary proof.

Let $\left(e_{1}, \ldots, e_{4}\right)$ be an orthonormal basis of $T_{x} S^{4}$ and for convenience set $R_{i j}^{ \pm}=R_{e_{l}, e_{j}}^{ \pm}$. To begin we consider the quantities

$$
C_{j k} \equiv\left[R_{j k}^{+}, R_{j k}^{-}\right]=C_{k j}
$$

for $j, k=1, \ldots, 4$. Since $\tau=0$ we know that $\sum_{j} C_{j k}=0$ for each $k$, i.e., we have that

$$
\left\{\begin{array}{l}
C_{12}+C_{13}+C_{14}=0 \\
C_{12}+C_{23}+C_{24}=0 \\
C_{13}+C_{23}+C_{34}=0 \\
C_{14}+C_{24}+C_{34}=0
\end{array} .\right.
$$

Using the fundamental identities

$$
R_{12}^{ \pm}= \pm R_{34}^{ \pm}, \quad R_{13}^{ \pm}= \pm R_{42}^{ \pm}, \quad R_{14}^{ \pm}= \pm R_{23}^{ \pm},
$$


we see that

$$
C_{12}=-C_{34}, \quad C_{13}=-C_{24}, \quad C_{14}=-C_{23} .
$$

Combining (7.17) and (7.19) shows that $C_{j k}=0$ for all $j, k$. Thus, using (7.18) we have proved that

$$
\begin{cases}{\left[R_{j k}^{+}, R_{j k}^{-}\right]=0} & \text { for all } j, k \\ {\left[R_{i j}^{+}, R_{k l}^{-}\right]=0} & \text { when } i, j, k, l \text { are distinct } .\end{cases}
$$

It remains only to show that

$$
\left[R_{i j}^{+}, R_{i k}^{-}\right]=0 \quad \text { when } i, j, k \text { are distinct. }
$$

It clearly suffices to establish $(7.21)$ for the case $(i, j, k)=(3,1,2)$ since we are free to permute our indices. Note that

$$
\begin{aligned}
\tau_{12} & =\left[R_{31}^{+}, R_{32}^{-}\right]+\left[R_{41}^{+}, R_{42}^{-}\right]=0 \\
\tau_{34} & =\left[R_{13}^{+}, R_{14}^{-}\right]+\left[R_{23}^{+}, R_{24}^{-}\right] \\
& =\left[R_{13}^{+},-R_{23}^{-}\right]+\left[R_{14}^{+}, R_{24}^{-}\right] \\
& =-\left[R_{31}^{+}, R_{32}^{-}\right]+\left[R_{41}^{+}, R_{42}^{-}\right]=0 .
\end{aligned}
$$

Consequently, $\left[R_{31}^{+}, R_{32}^{-}\right]=0$ and the proposition is proved.

The next step in our proof is the following observation.

(7.22) Lemma. Let $\mathfrak{a}^{+}$and $\mathfrak{a}^{-}$be subalgebras of a Lie algebra $\mathfrak{g}$ such that $\left[\mathfrak{a}^{+}, \mathfrak{a}^{-}\right]=0$. If $\mathfrak{g} \cong \mathfrak{s u}_{2}, \mathfrak{s u}_{3}$ or $\mathfrak{u}_{2}$, then either $\mathfrak{a}^{+}$or $\mathfrak{a}^{-}$is abelian.

Proof. In each case it is straightforward to check that the centralizer of any nonabelian subalgebra is abelian.

Note that if $\mathfrak{g}$ is the Lie algebra of $G_{2}$ or if $\operatorname{rank}(\mathfrak{g}) \geqq 3$ then Lemma (7.22) is false since $\mathfrak{g}$ contains an $\mathfrak{s o}_{4}$ subalgebra (see Sect. 8).

We now consider the $\mathfrak{g}_{E^{-}}$valued 4-tensors $C^{+}=\left[R^{+}, R^{+}\right]$and $C^{-}=\left[R^{-}, R^{-}\right]$ on $S^{4}$. (That is, $C_{\bar{X}, Y, Z, W}^{ \pm}=\left[R_{X, Y}^{ \pm}, R_{Z, W}^{ \pm}\right]$for $X, Y, Z, W \in T_{x} S^{4}$.) It follows from (7.16) and (7.22) that either $C^{+}$or $C^{-}$must vanish on some open subset $\mathcal{O} \subseteq S^{4}$. For the sake of argument, suppose $C^{+}$vanishes on $\mathcal{O}$. Now $C^{+}$is an algebraic function of a solution of the elliptic equation $\Delta R^{+}=0$, and the Aronszajn theorem on unique continuation of solutions to elliptic systems [1] applies to prove that $C^{+}=0$ on $S^{4}$.

We now observe that, since $\left[R^{+}, R^{-}\right]$and $\left[R^{+}, R^{+}\right]$both vanish on $S^{4}$, formula (7.15) [and also the Bochner-Weitzenböck formula (3.9)] for $R^{+}$becomes

$$
\nabla^{*} \nabla R^{+}+4 R^{+}=0
$$

Since $\nabla^{*} \nabla \geqq 0$ on $S^{4}$, this implies $R^{+} \equiv 0$. (The argument in the case that $C^{-}$ vanishes on $\mathcal{O}$ is completely analogous, and shows that $R^{-} \equiv 0$.) This completes the proof of Theorem 7.12. 


\section{Principal $\mathrm{SO}_{4}$-Bundles}

We now focus our attention on the interesting special case of $\mathrm{SO}_{4}$-bundles over a 4-manifold. Here there is an additional topological constraint on the Yang-Mills functional. There is also a generalization of the condition of self-duality which occurs if and only if the minimum values permitted by the topology are attained. We then extend Theorem (7.12) to this case; that is, we prove that any weakly stable Yang-Mills field with group $\mathrm{SO}_{4}$ on the 4-sphere satisfies this condition. Each $\mathrm{SO}_{4}$-bundle over $S^{4}$ carries a large family of these minimizing connections.

Let $M$ be a compact, oriented 4-dimensional riemannian manifold, and consider over $M$ an oriented 4-dimensional riemannian vector bundle $E$ (i.e., a bundle with structure group $\mathrm{SO}_{4}$ ). Then there are Hodge operators

$$
\text { *: } \Lambda^{2} T M \rightarrow \Lambda^{2} T M \text { and is: } \Lambda^{2} E \rightarrow \Lambda^{2} E
$$

with corresponding decompositions

$$
\Lambda^{2} T M=\Lambda^{+} T M \oplus \Lambda^{-} T M \text { and } \Lambda^{2} E=\Lambda^{+} E \oplus \Lambda^{-} E,
$$

where $T M$ denotes the tangent bundle of $M$ and $\Lambda^{ \pm}$denotes the \pm 1 eigenspace of the star-operator. For any $\mathrm{SO}_{4}$-connection $\nabla$ on $E$, the curvature $R^{V}$ can be considered as a bundle map

$$
R^{\nabla}: \Lambda^{2} T M \rightarrow \Lambda^{2} E .
$$

[Recall the isomorphism $\Lambda^{2} E \cong \mathfrak{s o}_{E}$ given by (2.34).]

It can therefore be decomposed as

$$
R^{\nabla}=R_{+}^{+}+R_{-}^{+}+R_{+}^{-}+R_{-}^{-}
$$

with respect to the splittings (8.1). Since these splittings are orthogonal, we can write the Yang-Mills integrand as

$$
\left\|R^{V}\right\|^{2}=\left\|R_{+}^{+}\right\|^{2}+\left\|R_{-}^{+}\right\|^{2}+\left\|R_{+}^{-}\right\|^{2}+\left\|R_{-}^{-}\right\|^{2} .
$$

Since $E$ is oriented and of dimension 4 over a 4-manifold, it has two independent characteristic invariants, its Pontrjagin number $p_{1}(E)$ (cf. Sect. 2), and its Euler number $\chi(E)$. By Chern-Weil theory these numbers can be computed by integrating certain universal polynomials in the components of the curvature tensor $R$. From these standard formulas it is a straightforward calculation to show that:

$$
p_{1}(E)=\frac{1}{4 \pi^{2}} \int_{M}\left\{\left\|R_{+}^{+}\right\|^{2}+\left\|R_{-}^{+}\right\|^{2}-\left\|R_{+}^{-}\right\|^{2}-\left\|R_{-}^{-}\right\|^{2}\right\}
$$

and

$$
\chi(E)=\frac{1}{8 \pi^{2}} \int_{M}\left\{\left\|R_{+}^{+}\right\|^{2}-\left\|R_{-}^{+}\right\|^{2}-\left\|R_{+}^{-}\right\|^{2}+\left\|R_{-}^{-}\right\|^{2}\right\} .
$$

Both of these numbers are integers, and both may be either positive or negative. From (8.3), (8.4), and (8.5) we immediately conclude the following.

(8.6) Theorem. Let $E$ be an oriented 4-dimensional riemannian vector bundle over a compact oriented 4-dimensional riemannian manifold $M$. Let $p=4 \pi^{2} p_{1}(E)$ and 
$\chi=8 \pi^{2} \chi(E)$ denote the (renormalized) Pontrjagin and Euler numbers of $E$ respectively. Then

$$
\mathscr{Y} \mathscr{M}(\nabla) \geqq \max (|p|,|\chi|)
$$

for any $\mathrm{SO}_{4}$-connection $\nabla$ on $E$. Furthermore this minimum value is attained if and only if :

$$
\left\{\begin{array}{ccc}
R_{-}^{-}=R_{+}^{-}=0 & \text { if } & p \geqq|\chi|, \\
R_{-}^{+}=R_{+}^{-}=0 & \text { if } & \chi \geqq|p|, \\
R_{-}^{+}=R_{+}^{+}=0 & \text { if } & -p \geqq|\chi|, \text { and } \\
R_{+}^{+}=R_{-}^{-}=0 & \text { if } & -\chi \geqq|p| .
\end{array}\right.
$$

For the sake of exposition we briefly present some examples.

(8.8) Example. Suppose the structure group of $E$ can be reduced to $\mathrm{SU}_{2}$. In this case $-\frac{1}{2} p_{1}(E)=\chi(E)=c_{2}(E)$, the second Chern class of $E$. Here Theorem (8.6) asserts that $\mathscr{Y} \mathscr{M}(\nabla)=4 \pi^{2}\left|p_{1}(E)\right|$, the minimum allowed value, if and only if

$$
R=R_{-}^{+} \quad \text { if } \quad p_{1}(E) \geqq 0
$$

and

$$
R=R_{-}^{-} \quad \text { if } \quad p_{1}(E) \leqq 0
$$

(8.9) Example. Let $M=S^{4}$ and let $E=T M$, the tangent bundle. Then $p_{1}(T M)=0$ and $\chi(T M)=2$. For the canonical riemannian connection, the curvature

$$
R: \Lambda^{2} T M \rightarrow \Lambda^{2} T M
$$

is the identity map. Hence,

$$
R_{-}^{+}=R_{+}^{-}=0
$$

and $R_{ \pm}^{ \pm}: \Lambda^{ \pm} T M \rightarrow \Lambda^{ \pm} T M$ is the identity map. (Thus $\left\|R_{+}^{+}\right\|^{2}=\left\|R_{-}^{-}\right\|^{2}=3$.) In particular, by Theorem (8.6) the Levi-Civita connection on $T^{4}$ achieves an absolute minimum of the Yang-Mills functional.

Recall that on a 4-manifold, a curvature tensor $R^{\nabla}$ is harmonic if and only if the components $R^{+}$and $R^{-}$are harmonic. We have the following analogue.

(8.10) Proposition. Let $E$ and $M$ be as in Theorem (8.6), and let $R^{\nabla}$ be the curvature of an $\mathrm{SO}_{4}$-connection $\nabla$ on $E$. Then $R^{\nabla}$ is harmonic (i.e., Yang-Mills) if and only if each of the components $R_{+}^{+}, R_{-}^{+}, R_{+}^{-}$, and $R_{-}^{-}$is harmonic.

Proof. If each component of $R^{\nabla}$ is harmonic, then clearly $R^{\nabla}$ is harmonic. On the other hand suppose $R^{\nabla}$ is harmonic. Then $R^{+}$and $R^{-}$are harmonic by (2.31). Furthermore, $R^{+}=R_{+}^{+}+R_{-}^{+}$and $R_{ \pm}^{+}=\frac{1}{2}(1 \pm \hat{\tau}) R^{+}$. The corresponding statement is true for $R^{-}$. Now the operator $\boldsymbol{\tau}^{2}: \Lambda^{2} E \rightarrow \Lambda^{2} E$ is parallel, i.e., $\nabla(\hat{\tau})=[\nabla, \hat{\tau}]=0$, since $\hat{r}$ is defined in terms of the metric on $E$. It follows directly from definitions that $d^{\nabla}$ and $\delta^{\nabla}$ (and therefore $\Delta^{\nabla}$ ) commute with $\boldsymbol{\tau}$. Thus $\Delta^{\nabla}\left(R_{ \pm}^{+}=\frac{1}{2}(1 \pm \hat{\xi}) \Delta^{\nabla} R^{+}=0\right.$ and $\Delta^{\nabla}\left(R_{ \pm}^{-}\right)=\frac{1}{2}\left(1 \pm \Delta^{\nabla}\right) \Delta^{-}=0$. This completes the proof. 
We are now ready to prove the main result of this section.

(8.11) Theorem. Any weakly stable Yang-Mills field on $\mathrm{S}^{4}$ with structure group $\mathrm{SO}_{4}$ satisfies the condition (8.7) of two-fold self-duality.

Proof. From the arguments of Sect. 7 [see Proposition (7.16)] we know that the endomorphisms $R_{.}^{+}$, and $R_{\cdot}^{-}$, commute at each point of $S^{4}$. We now observe that under the natural identification $\Lambda^{2} E \cong \mathfrak{s o}_{E}$, given by (2.34), the decomposition $\Lambda^{+} E \oplus \Lambda^{-} E$ corresponds to a decomposition

$$
\mathfrak{s o}_{E}=\mathfrak{s o}_{E}^{+} \oplus \mathfrak{s o}_{E}^{-},
$$

where $\mathfrak{s o}_{E}^{ \pm}$are bundles of sub-Lie algebras pointwise isomorphic to $\mathfrak{s u}_{2}$, and where

$$
\left[\mathfrak{s o}_{E}^{+}, \mathfrak{s o}_{E}^{-}\right] \equiv 0 .
$$

(This corresponds to the decomposition $\mathfrak{s o}_{4}=\mathfrak{s u}_{2} \oplus \mathfrak{s u}_{2}$.) Consequently, at each point of $S^{4}$ the endomorphisms $\left(R_{+}^{+}\right)_{,}, \ldots, \ldots,\left(R_{-}^{-}\right)_{.,}$mutually commute.

We now focus attention on the harmonic $\mathfrak{s o}_{E}^{+}$-valued tensor $R_{+}=R_{+}^{+}+R_{+}^{-}$. Since $\mathfrak{s o}_{E}^{+}$is a bundle of $\mathfrak{s u}_{2}$-algebras, the arguments of Sect. 7 can be applied directly to prove that either $R_{+}^{+}$or $R_{+}^{-}$vanishes on $S^{4}$. The same arguments applied to $R_{-}=R_{-}^{+}+R_{-}^{-}$prove that either $R_{-}^{+}$or $R_{-}^{-}$vanishes on $S^{4}$. Therefore we are in one of the four cases of condition (8.7). Which case or cases occur is now completely determined by the topology and the formulas (8.4) and (8.5). This completes the proof.

We now show that each $\mathrm{SO}_{4}$-bundle over $S^{4}$ carries a large family of connections satisfying condition (8.7). To begin we look at the topological classification of these bundles. Note that since $S^{4}$ is 2-connected, any $\mathrm{SO}_{4}$-bundle carries a unique spin structure, i.e., we may replace the principal $\mathrm{SO}_{4}$-bundle $P_{\mathrm{SO}_{4}}$ with a principal $\mathrm{Spin}_{4}$-bundle $P_{\mathrm{Spin}_{4}}$ and an equivalence $P_{\mathrm{Spin}_{4}} / \mathbb{Z}_{2} \cong P_{\mathrm{SO}_{4}}$. Since $\mathrm{Spin}_{4} \cong \mathrm{SU}_{2} \times \mathrm{SU}_{2}$, there is a splitting of $P_{\text {Spin }_{4}}$ into a Whitney sum

$$
P_{\mathrm{Spin}_{4}} \cong P_{\mathrm{SU}_{2}}^{\prime} \times P_{\mathrm{SU}_{2}}^{\prime \prime}
$$

of principal $\mathrm{SU}_{2}$-bundles. (Otherwise said, we lift the classifying map $S^{4} \rightarrow \mathrm{BSO}_{4}$ for $P_{\mathrm{SO}_{4}}$ to a map $S^{4} \rightarrow B \mathrm{Spin}_{4} \cong B \mathrm{SU}_{2} \times B \mathrm{SU}_{2}$.) Each of the bundles $P_{\mathrm{SU}_{2}}^{(k)}$ over $S^{4}$ is classified by its first Pontryagin number $p^{(k)} \in \mathbb{Z}$. There is exactly one such bundle, up to equivalence, for each integer. Consequently, the principal $\mathrm{SO}_{4}$-bundles over $S^{4}$, up to equivalence, are in one-to-one correspondence with pairs of integers $\left(p^{\prime}, p^{\prime \prime}\right)$ according to the prescription above.

Suppose $P_{\mathrm{SO}_{4}}$ corresponds to the pair $\left(p^{\prime}, p^{\prime \prime}\right)$ and let $E$ be the associated 4-plane bundle over $S^{4}$. Then it is not difficult to show that

$$
\begin{gathered}
p_{1}(E)=2\left(p^{\prime}+p^{\prime \prime}\right) \\
\chi(E)=p^{\prime}-p^{\prime \prime}
\end{gathered}
$$

We now recall that the principal $\mathrm{SU}_{2}$ bundle with Pontryagin number $p \neq 0$ carries an $(8|p|-3)$-dimensional family $\mathscr{M}_{p}$ of non-gauge-equivalent connections which are self-dual or anti-self-dual depending on whether $p$ is positive or negative $[2,3,9]$. (By convention, we let $\mathscr{M}_{0}$ denote the class of the flat connection of the 
trivial bundle.) Suppose then that $P_{\mathrm{Spin}_{4}}=P_{\mathrm{SU}_{2}}^{\prime} \times P_{\mathrm{SU}_{2}}^{\prime \prime}$ corresponds to the pair of integers $\left(p^{\prime}, p^{\prime \prime}\right)$. Choose connections $\nabla^{(j)} \in \mathscr{M}_{p^{(j)}}$ and introduce the direct sum connection $\nabla^{\prime} \oplus \nabla^{\prime \prime}$ on $P_{\mathrm{SU}_{2}}^{\prime} \times P_{\mathrm{SU}_{2}}^{\prime \prime}$. This descends to a connection on $P_{\mathrm{SO}_{4}}$ and on its associated 4-plane bundle $E$. The components $R_{+}$and $R_{-}$of the curvature of this connection correspond exactly to the curvature tensors of $P_{\mathrm{SU}_{2}}^{\prime}$ and $P_{\mathrm{SU}_{2}}^{\prime \prime}$ respectively. Consequently, this connection satisfies (8.7). We have proved the following.

(8.13) Theorem. Let $E$ be an oriented riemannian 4-plane bundle over $S^{4}$, and set $p=\frac{1}{2} p_{1}(E)$ and $\chi=\chi(E)$. Then there is a family $\mathscr{M}$ of gauge-inequivalent connections satisfying (8.7) on E where

$$
\operatorname{dim}(\mathscr{M})=\left\{\begin{array}{lll}
4(|p+\chi|+|p-\chi|)-6 & \text { if } & p \neq \pm \chi \\
4(|p+\chi|+|p-\chi|)-3 & \text { if } & p= \pm \chi \neq 0 \\
0 & \text { if } & p=\chi=0
\end{array}\right.
$$

Given a riemannian 4-plane bundle $E$ over $S^{4}$, we now consider the bundle $S_{E}$ of unit spheres in the fibres of $E$. The total space of $S_{E}$ is homeomorphic to $S^{7}$ if and only if $\chi(E)=1$. However, $S_{E}$ is diffeomorphic to $S^{7}$ only if, in addition, we have a condition on $p_{1}(E)\left(\left(p_{1}(E)\right)^{2} \equiv 4 \bmod 27.7\right.$, see Milnor [12]). In any case, a riemannian connection on $E$ induces a field of "horizontal" planes on the manifold $S_{E}$, i.e., a field of 4-planes $\tau$ everywhere transverse to the fibres. We lift the metric of $S^{4}$ to $\tau$ via the bundle projection $\pi: S_{E} \rightarrow S^{4}$; we introduce the obvious euclidean metric (induced from the inner product in $E$ ) on the fibres; and we declare $\tau$ to be orthogonal to the tangent spaces to the fibres. This gives a metric on $S_{E}$ defined canonically in terms of the connection on $E$. This metric always has positive Ricci curvature. However, one can show, by building on arguments of Weinstein [17], that these metrics are never of positive sectional curvature.

\section{The Stability of $T\left(S^{n} / \Gamma\right)$}

We have shown in Sect. 7 that any Yang-Mills field on $S^{n}, n \geqq 5$, is unstable. This applies in particular to the Levi-Civita connection on the tangent bundle $T S^{n}$ which is clearly Yang-Mills since $D R \equiv 0$. In contrast to this we now prove the following result.

(9.1) Theorem. Let $\Gamma$ be a non-trivial, finite group of isometries acting freely on $S^{n}$, $n \geqq 4$, and consider the quotient manifold $S^{n} / \Gamma$ with its "quotient" metric of constant sectional curvature. Then the Levi-Civita connection on $T\left(S^{n} / \Gamma\right)$, considered as a Yang-Mills field, is strictly stable.

We know that the standard connection on $T S^{4}$ is weakly stable. This follows directly from the fact that on $T S^{4}$ this connection actually minimizes the YangMills functional [see (8.9)]. One wonders whether the Levi-Civita connection is minimizing in all the cases $T\left(S^{n} / \Gamma\right)$ of Theorem $(9.1)$.

We shall also prove the following.

(9.2) Theorem. The Levi-Civita connection on $T S^{3}$, or on $T\left(S^{3} / \Gamma\right)$ for any $\Gamma$ as above, is unstable as a Yang-Mills field. In fact its index is 1 and its nullity is 0. 
Note that since $T\left(S^{3} / \Gamma\right)$ is topologically trivial, (note that $S^{3} / \Gamma$ is orientable since $\Gamma$ acts without fixed points) the minimum of the Yang-Mills functional in this case is zero and is achieved by the flat connections.

We point out that recently Uhlenbeck has proved the weak compactness theorem necessary to do Morse theory for $\mathscr{Y} \mathscr{M}: \mathscr{C}_{T} / \mathscr{G}_{T} \rightarrow \mathbb{R}$ in the case of 3-manifolds.

Both Theorems (9.1) and (9.2) will be proved by explicitly computing the operator

$$
\mathscr{S}=D^{*} D+(n-1) I+2 \mathfrak{R}
$$

on $\Omega^{1}\left(\mathfrak{s o}_{T}\right)$ (see Sect. 6). Note that $\Omega^{1}\left(\mathfrak{s o}_{T}\right)$ is just the space of smooth sections of the bundle $T^{*} \otimes \mathfrak{s o}_{T}$, where $T=T\left(S^{n} / \Gamma\right)$ and where $\mathfrak{s o}_{T} \cong \Lambda^{2} T$.

(9.4) Proposition. There is a natural orthogonal decomposition

$$
T^{*} \otimes \mathfrak{s o}_{T}=F_{1} \oplus F_{2} \oplus F_{3},
$$

where $F_{1} \cong T^{*}$ and $F_{2} \cong \Lambda^{3} T^{*}$, such that $\mathscr{S}\left(\Gamma\left(F_{j}\right)\right) \cong \Gamma\left(F_{j}\right)$ for each $j$. Furthermore,

$$
\begin{array}{llll}
\mathscr{S}=D^{*} D-(n-3) & \text { on } & \Gamma\left(F_{1}\right), \\
\mathscr{S}=D^{*} D+(n-5) & \text { on } & \Gamma\left(F_{2}\right), \\
\mathscr{S}=D^{*} D+(n+1) & \text { on } & \Gamma\left(F_{3}\right) .
\end{array}
$$

Proof. We shall work at a point $x \in S^{n}$. For $B \in T^{*} \otimes \mathfrak{s v}_{T_{x}}$ and $X \in T$, we let $B_{X}: T \rightarrow T$ denote the corresponding skew-symmetric linear map. For convenience we set $F=T^{*} \otimes \mathfrak{s o}_{T}$. Then the decomposition of $F$ at $x$ is given as follows :

$$
\begin{aligned}
& F_{1} \equiv\left\{B \in F: \exists V \in T \text { such that } B_{X}=X \wedge V\right\} \\
& F_{2} \equiv\left\{B \in F: B_{X}(Y)=-B_{Y}(X) \text { for all } X, Y \in T\right\} \\
& F_{3} \equiv \text { The orthogonal complement of } F_{1} \oplus F_{2} \text { in } F .
\end{aligned}
$$

The map $X \wedge V$ is defined by setting $(X \wedge V)(Y) \equiv\langle X, Y\rangle V-\langle V, Y\rangle X$. Observe that $F_{1} \perp F_{2}$, since for $B^{(k)} \in F_{k}$ we have

$$
\begin{aligned}
\left\langle B^{\prime}, B^{\prime \prime}\right\rangle & =\sum_{i} \sum_{j<k}\left\langle B_{e_{i}}^{\prime}\left(e_{j}\right), e_{k}\right\rangle\left\langle B_{e_{i}}^{\prime \prime}\left(e_{j}\right), e_{k}\right\rangle \\
& =\frac{1}{2} \sum_{i, j}\left\langle\left(e_{i} \wedge V\right)\left(e_{j}\right), B_{e_{i}}^{\prime \prime}\left(e_{j}\right)\right\rangle \\
& =\frac{1}{2} \sum_{i, j}\left\{\left\langle e_{i}, e_{j}\right\rangle\left\langle V, B_{e_{i}}^{\prime \prime}\left(e_{j}\right)\right\rangle+\left\langle V, e_{j}\right\rangle\left\langle B_{e_{j}}^{\prime \prime}\left(e_{i}\right), e_{i}\right\rangle\right\} .
\end{aligned}
$$

Similar calculations show that

$$
\left.F_{3}=\left\{B \in F: \sum_{j=1}^{n} B_{e_{j}} e_{j}=0 \text { and }\left\langle B_{X} Y, Z\right\rangle+\left\langle B_{Y} Z, X\right\rangle+\left\langle B_{Z} X, Y\right\rangle=0 \text { for all } X, Y\right\rangle\right\}
$$

(Note that we have dropped the parentheses for $B$.)

We now have an orthogonal decomposition $F=F_{1} \oplus F_{2} \oplus F_{3}$. The map

$$
T \approx F_{1}
$$

defined by $V \mapsto B^{V}$, where $\left(B^{V}\right)_{X}=X \wedge V$, is a bundle isomorphism. Furthermore, one easily sees that $D\left(B^{V}\right)=B^{(D V)}$, i.e., this isomorphism is connection preserving. 
Similarly we have that the map

$$
\Lambda^{3} T^{*} \rightarrow F_{2}
$$

defined by $\varphi \mapsto B^{\varphi}$, where $\left\langle B_{X}^{\varphi} Y, Z\right\rangle=\varphi(X, Y, Z)$, is a connection-preserving bundle isomorphism. This proves the following fact.

(9.7) Lemma. Each of the subbundles $F_{j}, j=1,2,3$, is preserved by the connection. In particular,

$$
D^{*} D: \Gamma\left(F_{j}\right) \rightarrow \Gamma\left(F_{j}\right)
$$

for each $j$. Moreover, for $j=1$ and 2, the bundle isomorphisms (9.5) and (9.6) take this operator to the corresponding operator $D^{*} D$ defined in terms of the Levi-Civita connection on $T \cong T^{*}$ and $\Lambda^{3} T^{*}$ respectively.

We must now examine the operator $\mathfrak{R}$. Recall that on $S^{n} / \Gamma$ the curvature tensor of $T$, considered as an element in $\Lambda^{2} T^{*} \otimes \mathfrak{s o}_{T}$, is given by

$$
R_{X, Y}=X \wedge Y \text {. }
$$

Consequently, for $X, Y \in T$ and $B \in F$ we have

$$
\begin{aligned}
\mathfrak{R}(B)_{X}(Y)= & \sum_{j=1}^{n}\left[R_{e_{j}, X}, B_{e_{j}}\right](Y) \\
= & -\sum_{j=1}^{n}\left[e_{j} \wedge X, B_{e_{j}}\right](Y) \\
= & -\sum_{j=1}^{n}\left\{\left(e_{j} \wedge X\right)\left(B_{e_{j}} Y\right)-B_{e_{j}}\left(\left(e_{j} \wedge X\right)(Y)\right)\right\} \\
= & \sum_{j=1}^{n}\left\{\left\langle X, B_{e_{j}} Y\right\rangle e_{j}-\left\langle e_{j}, B_{e_{j}} Y\right\rangle X\right. \\
& \left.-\langle X, Y\rangle B_{e_{j}} e_{j}+\left\langle e_{j}, Y\right\rangle B_{e_{j}} X\right\} \\
= & \sum_{j=1}^{n}\left\{e_{j} \wedge B_{e_{j}} X+B_{e_{j}} e_{j} \wedge X\right\}(Y) .
\end{aligned}
$$

Case 1. Suppose $B \in F_{1}$, that is, $B_{X}=X \wedge V$ for some $V \in T$. Then

$$
\sum B_{e_{j}} e_{j}=\sum\left(e_{j} \wedge V\right)\left(e_{j}\right)=\sum\left\{\left|e_{j}\right|^{2} V-\left\langle V, e_{j}\right\rangle e_{j}\right\}=(n-1) V,
$$

and so $\sum B_{e_{j}} e_{j} \wedge X=(n-1) V \wedge X=-(n-1) B_{X}$. On the other hand,

$$
\begin{aligned}
\sum_{j}\left(e_{j} \wedge B_{e_{j}} X\right)(Y) & =\sum_{j}\left\{\left\langle e_{j}, Y\right\rangle B_{e_{j}} X-\left\langle B_{e_{j}} X, Y\right\rangle e_{j}\right\} \\
& =B_{Y} X-\sum_{j}\left\langle\left(e_{j} \wedge V\right)(X), Y\right\rangle e_{j} \\
& =(Y \wedge V)(X)-\sum_{j}\left\{\left\langle e_{j}, X\right\rangle\langle V, Y\rangle-\langle V, X\rangle\left\langle e_{j}, Y\right\rangle\right\} e_{j} \\
& =\langle Y, X\rangle V-\langle V, X\rangle Y-\langle V, Y\rangle X+\langle V, X\rangle Y \\
& =(X \wedge V)(Y) \\
& =B_{X} Y .
\end{aligned}
$$

Consequently, $\mathfrak{R}(B)=-(n-2) B$. 
Case 2. Suppose $B \in F_{2}$, that is, $B_{X} Y=-B_{Y} X$ for all $X, Y$. Then clearly $\sum B_{e_{j}} e_{j}=0$, and so

$$
\begin{aligned}
\mathfrak{R}(B)_{X}(Y) & =\sum\left\{\left\langle e_{j}, Y\right\rangle B_{e_{j}} X-\left\langle B_{e_{j}} X, Y\right\rangle e_{j}\right\} \\
& =B_{Y} X+\sum\left\langle B_{X} e_{j}, Y\right\rangle e_{j} \\
& =-B_{X} Y-\sum\left\langle B_{X} Y, e_{j}\right\rangle e_{j} \\
& =-2 B_{X} Y .
\end{aligned}
$$

Case 3. Suppose $B \in F_{3}$, that is, suppose $\sum_{j} B_{e_{j}} e_{j}=0$ and

$$
\left\langle B_{X} Y, Z\right\rangle+\left\langle B_{Y} Z, X\right\rangle+\left\langle B_{Z} X, Y\right\rangle=0
$$

for all $X, Y, Z$. Then as in Case 2,

$$
\begin{aligned}
\mathfrak{R}(B)_{X}(Y) & =B_{Y} X-\sum_{j}\left\langle B_{e_{j}} X, Y\right\rangle e_{j} \\
& =B_{Y} X+\sum_{j}\left\{\left\langle B_{X} Y, e_{j}\right\rangle+\left\langle B_{Y} e_{j}, X\right\rangle\right\} e_{j} \\
& =B_{X} Y .
\end{aligned}
$$

Summarizing, we have that

$$
\mathfrak{R}=\left\{\begin{array}{lll}
-(n-2) & \text { on } & F_{1} \\
-2 & \text { on } & F_{2} \\
1 & \text { on } & F_{3} .
\end{array}\right.
$$

Combining (9.11) with (9.3) completes the proof of Proposition (9.4).

Proof of Theorem (9.1). From Proposition (9.4) and Lemma (9.7) we conclude that the Levi-Civita connection on $T=T\left(S^{n} / \Gamma\right)$ is strictly stable if

$$
D^{*} D>(n-3) \text { on } T^{*}
$$

and

$$
D^{*} D>-(n-5) \text { on } \Lambda^{3} T^{*},
$$

where $D^{*} D$ is defined using the natural extension of the Levi-Civita connection. Note that condition (9.13) is satisfied whenever $n \geqq 5$. (When $n=5$, we observe that $D^{*} D>0$ since its kernel consists of parallel 3-forms.) On $T^{*}$ we have that $\Delta=D^{*} D$ $+\mathrm{Ric}=D^{*} D+(n-1)$ where $\Delta=d \delta+\delta d$ is the Hodge Laplacian. Consequently, for $n \geqq 5$ we have stability if

$$
\Delta>2(n-2) \text { on } T^{*} .
$$

Let $\lambda_{k}\left(S^{n} / \Gamma\right)$ denote the $k^{\text {th }}$ eigenvalue of $\Delta$ on $T^{*}$. Then $\lambda_{1}\left(S^{n} / \Gamma\right) \geqq \lambda_{2}\left(S^{n}\right)$ if $\Gamma$ is nontrivial, since the first eigenspace of $\Delta$ on $T\left(S^{n}\right) \cong T^{*}\left(S^{n}\right)$ is exactly the space $\mathscr{V}$ defined in Sect. 7 and no element of $\mathscr{V}$ is $\Gamma$-invariant. One computes easily from [4] that $\lambda_{2}\left(S^{n}\right)=2(n-1)$. This proves Theorem (9.1) for $n \geqq 5$.

When $n=4$ we have a connection preserving bundle isometry $*: T^{*} \rightarrow \Lambda^{3} T^{*}$, and conditions (9.12) and (9.13) coincide. The argument in the paragraph above shows that $D^{*} D \geqq 3$, and the proof is complete. 
Proof of Theorem (9.2). Since $n=3$ and $D^{*} D \geqq 1$ on $T$ we know from Proposition (9.4) that $\mathscr{S}>0$ on $F_{1}$ and $F_{3}$. The bundle $F_{2} \cong \Lambda^{3} T^{*}$ is trivial and $D^{*} D$ is equivalent to the standard Laplace Beltrami operator on functions. Its first non-zero eigenvalue is $\geqq 3$. However, it has a 1 -dimensional null space generated by the volume form $* 1$. This corresponds to the element $B \in T^{*} \otimes \mathfrak{s o}_{T}$ defined by setting

$$
B_{X}(Y)=*(X \wedge Y)
$$

where $*: \Lambda^{2} T \rightarrow T$ is the Hodge star operator. Clearly $D B=0$, so, in particular, $\delta B=0$. From (9.4) we know that

$$
\mathscr{S}(B)=-2 B .
$$

Since the next eigenvalue of $D^{*} D$ on $F_{2}$ is $\geqq 3$, we know that $\mathscr{S}>0$ on the orthogonal complement of $B$. This completes the proof.

\section{Results on General Homogeneous Spaces}

Many of the ideas developed in Sects. 6 and 7 for $S^{n}$ can be carried over to any homogeneous riemannian manifold. This contradicts the long-standing belief of the authors that the noncompactness of the conformal group of the sphere was crucial in the proof of the Stability theorems. We begin by proving the following generalization of our fundamental result, Theorem (7.12).

(10.1) Theorem. Let $M=K / H$ be a compact orientable homogeneous riemannian manifold of dimension 4. Then any weakly stable Yang-Mills field over $M$ with group $\mathrm{SU}_{2}$, is either self-dual, or anti-self-dual, or reduces to an abelian field.

Proof. Let $K$ be the group of isometries of $M$ and let $\kappa$ be its associated Lie algebra of Killing vector fields. There exists on $\kappa$ a $K$-invariant inner product such that for each point $x \in M$, the natural map

$$
\kappa \stackrel{\varepsilon_{x}}{\longrightarrow} T_{x} M
$$

defined by $\varepsilon_{x}(V)=V_{x}$, is an isometry on $\underline{\operatorname{ker}}\left(\varepsilon_{x}\right)^{\perp}$. We fix this inner product on $\kappa$.

Consider now a weakly stable Yang-Mills field $(E, P, G, \nabla)$ over $M$, and let $\varphi \in \Omega^{2}\left(\mathfrak{g}_{E}\right)$ be a $\mathfrak{g}_{E}$-valued 2-form. Then we defined a quadratic form $Q_{\varphi} \geqq 0$ on $\kappa$ as in Theorem (7.7). By formula (6.5) we know that

$$
Q_{\varphi}(V)=\int_{M}\left\langle\dot{\mathscr{S}}\left(i_{V} \varphi\right), i_{V} \varphi\right\rangle
$$

for $V \in \kappa$, where

$$
\stackrel{\mathscr{S}}{ }^{\nabla}=\delta^{\nabla} d^{\nabla}+\mathfrak{\Re}^{\nabla}
$$

The key fact is the following lemma whose proof we shall postpone.

(10.4) Lemma. If $\varphi$ is harmonic and if $V$ is a Killing vector field, then

$$
\dot{\mathscr{S}}^{\nabla}\left(i_{V} \varphi\right)_{X}=-\sum_{j}\left\{\left[R_{e_{j}, V}^{\nabla}, \varphi_{e_{j}, X}\right]+\left[R_{e_{j}, X}^{\nabla}, \varphi_{e_{j}, V}\right]\right\} .
$$


This lemma is valid in general dimensions. In dimension 4, we can choose our harmonic form to be $\varphi=R^{+}$. Then, writing $R^{\nabla}=R^{+}+R^{-}$, we see that

$$
\mathscr{\mathscr { S }}^{V}\left(i_{V} R^{+}\right)_{X}=-2 \sum_{j}\left[R_{e_{j}, V}^{-}, R_{e_{j}, X}^{+}\right] .
$$

We now take the trace of $Q_{\varphi}$. We move the trace under the integral sign and use the fact that for each point $x \in M$, we can choose an orthonormal basis $\left(V_{1}, \ldots, V_{N}\right)$ of $\kappa$ such that

$$
V_{1}(x)=e_{1}, \ldots, V_{4}(x)=e_{4}, \quad V_{5}(x)=\ldots=V_{N}(x)=0,
$$

where $\left(e_{1}, \ldots, e_{4}\right)$ is an orthonormal basis of $T_{x} M$. This gives

$$
\operatorname{trace}\left(Q_{\varphi}\right)=-2 \int_{M} \sum_{i, j, k}\left\langle\left[R_{e_{i}, e_{j}}^{-}, R_{e_{i}, e_{k}}^{+}\right], R_{e_{j}, e_{k}}^{+}\right\rangle .
$$

We know from Sect. 4 that the right side of (10.5) is symmetric in $X$ and $V$. Consequently, trace $\left(Q_{\varphi}\right)=0$, and since $Q_{\varphi} \geqq 0$ on $\kappa$ (because the field is weakly stable), this means $Q_{\varphi}=0$ on $\kappa$. Finally, since the field is weakly stable, this means $\stackrel{\stackrel{\mathscr{S}}{ }}{ }^{\nabla}\left(i_{V} R^{+}\right)=0$ for all $V \in \kappa$, i.e.,

$$
\sum_{j=1}^{4}\left[R_{e_{j}, Y}^{-}, R_{e_{j}, X}^{+}\right]=0
$$

for all tangent vector fields $X, Y$ on $M$. This was precisely the hypothesis of Proposition (7.16). We conclude that $\left[\mathfrak{a}^{+}, \mathfrak{a}^{-}\right] \equiv 0$, where at each point $x \in M, \mathfrak{a}_{x}^{ \pm}$is the sub-Lie algebra of $\left(\mathfrak{g}_{E}\right)_{x}$ generated by the transformations $R_{X, Y}^{ \pm}$for $X, Y \in T_{x} M$. Consequently, by arguing as in Sect. 7 we conclude that either $\left[\mathfrak{a}^{+}, \mathfrak{a}^{+}\right] \equiv 0$ or $\left[\mathfrak{a}^{-}, \mathfrak{a}^{-}\right] \equiv 0$ on $M$. In fact, from the elementary properties of the Lie algebras we know the following.

Suppose $G=\mathrm{SU}_{2}$, and suppose that both $\mathfrak{a}^{+}$and $\mathfrak{a}^{-}$are not zero. Then as we saw in Sect. 7, neither can vanish on an open subset of $M$. Consequently, both $\mathfrak{a}^{+}$ and $\mathfrak{a}^{-}$are abelian at each point. Since $\left[\mathfrak{a}^{+}, \mathfrak{a}^{-}\right]=0$, we conclude that $\mathfrak{a}=\mathfrak{a}^{+}+\mathfrak{a}^{-}$ is also abelian everywhere on $M$. In particular, $\operatorname{dim} \mathfrak{a} \leqq 1$ everywhere on $M$. If $R^{\nabla}$ is not identically zero, then an open dense subset of $M$ we can write

$$
R_{X, Y}^{\nabla}=\varphi_{X, Y} \otimes \sigma,
$$

where $\varphi$ is a scalar 2-form and $\sigma$ is a section of $\mathfrak{g}_{E}$ of unit length at each point. Proposition (3.15) now implies that $\sigma$ is parallel and $\varphi$ is harmonic. On a compact 4-dimensional homogeneous space, every harmonic 2-form is parallel. (Check case-by-case.) Hence $\sigma$ is a globally defined, parallel section of $\mathfrak{g}_{e}$. At each point $p \in M, \sigma_{p}$ is an endomorphism of the fibre $E_{p} \cong \mathbb{C}^{2}$ with eigenvalues $\lambda$ and $-\lambda$. (These eigenvalues are non-zero and constant on $M$.) The corresponding eigenbundle decomposition $E=E_{\lambda} \oplus E_{-\lambda}$ is parallel, i.e., is preserved by the connection. This gives the desired reduction to a $U_{1}$-structure group, i.e., to an abelian field.

Proof of Lemma (10.4). Fix a point $x \in M$, choose $X, e_{1}, \ldots, e_{4} \in T_{x} M$ so that $\left(e_{1}, \ldots, e_{4}\right)$ is an orthonormal basis. Extend $X$ to a local vector field and extend $e_{1}, \ldots, e_{4}$ to a local orthonormal frame field so that

$$
(D X)(x)=\left(D e_{1}\right)(x)=\ldots=\left(D e_{4}\right)(x)=0 .
$$


Since $\varphi$ is harmonic we have

$$
\begin{aligned}
\left(\delta^{\nabla} \varphi\right)_{X} & =-\sum\left(\nabla_{e_{j}} \varphi\right)_{e_{j}, X}=0 \\
\left(d^{\nabla} \varphi\right)_{X, Y, Z} & =\left(\nabla_{X} \varphi\right)_{Y, Z}+\left(\nabla_{Y} \varphi\right)_{Z, X}+\left(\nabla_{Z} \varphi\right)_{X, Y}=0
\end{aligned}
$$

for all $X, Y, Z$. Set $\hat{\varphi}=i_{V} \varphi$. Then at the point $x$,

$$
\begin{aligned}
\left(\delta^{\nabla} f^{\nabla} \hat{\varphi}\right)_{X}= & -\sum_{j}\left(\nabla_{e_{j}} d \hat{\varphi}\right) e_{j, X} \\
= & -\sum_{j}\left\{\nabla_{e_{j}}\left(d \hat{\varphi}_{e_{j}, X}\right)-d \hat{\varphi}_{D_{e_{j}}, X}-d \hat{\varphi}_{e D_{e} X}\right\} \\
= & -\sum_{j} \nabla_{e_{j}}\left\{\left(\nabla_{e_{j}} \hat{\varphi}\right)_{X}-\left(\nabla_{X} \hat{\varphi}\right)_{e_{j}}\right\} \\
= & -\sum_{j} \nabla_{e_{j}}\left\{\nabla_{e_{j}}\left(\hat{\varphi}_{X}\right)-\hat{\varphi}_{D_{e_{j}} X}-\nabla_{X}\left(\hat{\varphi}_{e_{j}}\right)+\hat{\varphi}_{D_{X} e_{j}}\right\} \\
= & -\sum_{j} \nabla_{e_{j}}\left\{\nabla_{e_{j}}\left(\varphi_{V, X}\right)-\varphi_{V, D_{e_{j}} X}-\nabla_{X}\left(\varphi_{V, e_{j}}\right)+\varphi_{V, D_{X} e_{j}}\right\} \\
= & -\sum_{j} \nabla_{e_{j}}\left\{\left(\nabla_{e_{j}} \varphi\right)_{V, X}+\varphi_{D_{e^{\prime}} V, X}-\left(\nabla_{X} \varphi\right)_{V, e_{j}}-\varphi_{D_{X} V, e_{j}}\right\} \\
= & -\sum_{j} \nabla_{e_{j}}\left\{\left(\nabla_{V} \varphi\right)_{e_{j}, X}+\varphi_{D_{e_{j}} V, X}-\varphi_{D_{X} V, e_{j}}\right\} \\
= & -\sum_{j}\left\{\left(\nabla_{e_{j}}\left(\nabla_{V} \varphi\right)\right)_{e_{j}, X}+\left(\nabla_{e_{j}} \varphi\right)_{D_{e_{j}} V, X}+\varphi_{D_{e_{j}, e_{j}}^{2} V, X}\right. \\
& \left.-\left(\nabla_{e_{j}} \varphi\right)_{X} V, e_{j}-\varphi_{D_{e_{j}}^{2}, X} V, e_{j}\right\} \\
= & -\sum_{j}\left\{\left(\nabla_{e_{j}, V}^{2} \varphi\right)_{e_{j}, X}+\left(\nabla_{D_{e_{j}} V} \varphi\right)_{e_{j}, X}+\left(\nabla_{e_{j}} \varphi\right)_{D_{e_{j}} V, X}\right. \\
& \left.+\varphi_{D_{e_{j}, e_{j} V, X}^{2} V}-\varphi_{D_{e_{j}, X}^{2} V, e_{j}}\right\} .
\end{aligned}
$$

Since $\delta^{\nabla} \varphi=0$ and $R_{X, Y}=\nabla_{X, Y}^{2}-\nabla_{Y, X}^{2}$, we have

$$
\begin{aligned}
\sum_{j}\left(\nabla_{e_{j}, V}^{2} \varphi\right)_{e_{j}, X} & =\sum_{j}\left(R_{e_{j}, V}^{\nabla} \varphi\right)_{e_{j}, X} \\
& =\sum_{j}\left\{\left[R_{e_{j}, V}^{\nabla}, \varphi_{e_{j}, X}\right]-\varphi_{R_{e_{j}, V} e_{j}, X}-\varphi_{e_{j}, R_{e_{j}, V} X}\right\} .
\end{aligned}
$$

Furthermore since $V$ is a Killing field it satisfies the following equations:

$$
\begin{aligned}
D_{e_{j}, X}^{2} V & =R_{e_{j}, V} X \\
D^{*} D V & =-\sum_{j} R_{e_{j}, V} e_{j}=\operatorname{Ric}(V) .
\end{aligned}
$$

Substituting these last three equations back into (10.7) gives:

$$
\left(\delta^{\nabla} d^{\nabla} \hat{\varphi}\right)_{X}=-\sum_{j}\left[R_{e_{j}, V}^{\nabla}, \varphi_{e_{j}, X}\right]-\sum_{j}\left[\left(\nabla_{D_{e_{j} V}} \varphi\right)_{e_{j}, X}+\left(\nabla_{e_{j}} \varphi\right)_{D_{e_{j}, V}, X}\right\} .
$$

A Killing vector field $V$ has the fundamental property that $\left\langle D_{X} V, Y\right\rangle=-\left\langle D_{Y} V, X\right\rangle$ for all $X, Y$. Writing $D_{e_{j}} V=\sum_{k} a_{j k} e_{k}$ we have that $a_{j k}=-a_{k j}$. Substituting this into 
the second summation in (10.8) gives:

$$
\sum_{j, k}\left\{a_{j k}\left(\nabla_{e_{k}} \varphi\right)_{e_{j}, X}+a_{j k}\left(\nabla_{e_{j}} \varphi\right)_{e_{k}, X}\right\}=0 .
$$

Consequently, we have that

$$
\left(\delta^{\nabla} d^{\nabla} \hat{\varphi}\right)_{X}=-\sum_{j}\left[R_{e_{j}, V}^{\nabla}, \varphi_{e_{j}, X}\right] .
$$

By definition we know that

$$
\mathfrak{R}^{\nabla}(\hat{\varphi})_{X}=\sum_{j}\left[R_{e_{j}, X}^{\nabla}, \hat{\varphi}_{e_{j}}\right]=-\sum_{j}\left[R_{e_{j}, X}^{\nabla}, \varphi_{e_{j}, V}\right] .
$$

Adding these last two equations proves the lemma.

(10.9) Remark. Theorem (10.1) can be easily generalized to fields with group $U_{2}$, $\mathrm{SU}_{3}$ or $\mathrm{SO}_{4}$. A detailed statement will appear in a forthcoming note by the authors (to appear in Annals of Math. Studies volume edited by S. T. Yau).

Acknowledgements. We would like to thank I.H.E.S. and the Volkswagen Foundation for supporting the research of the second author. We would also like to acknowledge the tremendous importance to this work of the meeting, "Journées Yang-Mills", which was sponsored by the Société Mathématique de France in Paris in May, 1978. Finally, and above all, we want to thank Jim Simons whose ideas were the fundamental inspiration of this paper.

\section{References}

1. Aronszajn, N.: J. Math. Pure Appl. 35, 235-249 (1957)

2. Atiyah, M.F., Drinfeld, V.G., Hitchin, N.J., Manin, Y.I.: Phys. Lett. A65, 185-187 (1978)

3. Atiyah, M.F., Hitchin, N.J., Singer, I.M. : Proc. R. Soc. London Ser. A362, 425-461 (1978)

4. Besse, A. : Manifolds all of whose geodesics are closed. Berlin, Heidelberg, New York : Springer 1977

5. Bourguignon, J.-P.: Séminaire A. Besse sur la géométrie riemannienne de dimension 4. Expos. $n^{\circ}$ XVI, 1-26 (to appear)

6. Bourguignon, J.-P., Karcher, H.: Ann. Sci. E.N.S. 11, 71-92 (1978)

7. Bourguignon, J.-P., Lawson, Jr., H.B., Simons, J.: Proc. Acad. Sci. USA 76, 1550-1553 (1979)

8. Daniel, M., Mitter, P.K., Viallet, C.M.: Phys. Lett. B77, 77-79 (1978)

9. Drinfeld, V.G., Manin, Y.I.: Commun. Math. Phys. 63, 177-192 (1978)

10. Gallot, S., Meyer, D.: J. Math. Pures Appl. 54, 259-284 (1975)

11. Gantmacher, F.R.: The theory of matrices. New York: Chelsea Press, N.Y. 1959

12. Milnor, J.: Ann. Math. 64, 399-405 (1956)

13. Milnor, J.: Morse theory. Annals of Math. Studies, No. 51. Princeton, NJ : Princeton University Press 1963

14. Palais, R.S., Smale, S.: Bull. A.M.S. 70, 165-171 (1964)

15. Thurston, W.: Bull. A.M.S. 80, 304-307 (1974)

16. Uhlenbeck, K.: To appear

17. Weinstein, A.: Fat bundles. Preprint 1980

Communicated by A. Jaffe

Received March 7, 1980

Note added in proof. The result obtained by N. Hitchin on half conformally flat Einstein manifolds with positive scalar curvature has been obtained independently by T. Friedrich and H. Kurke from Berlin (GDR). 